Bull. Fac. Agric., Cairo Univ., 66: 156-170 (2015).

\title{
EFFECT OF MAGNETICALLY TREATED WATER AND METHODS OF TRAINING ON SOME VEGETATIVE GROWTH AND FLOWERING CHARACTERISTICS OF THREE GENOTYPES OF MUSKMELON (Cucumis melo) UNDER GREENHOUSE CONDITION
}

(Received:16.6.2015)

By

\author{
A. M. A. Al-Shammary and R . H. Asmaael
}

Department of Horticulture \& Landscape, College of Agriculture, University of Diyala, Iraq

\begin{abstract}
The current study was conducted in the new nursery of New Baquba / Diyala agriculture office during the growing season 2014, to study the effect of magnetically treated water and methods of training on the characteristics of the vegetative and flowering growth for three muskmelon genotypes. The experiment included 24 treatments as combinations between the three muskmelon genotypes (RAND, NADA and IDEAL), two types of water (natural water and magnetically treated water at 3000 gauss) and four training methods (one stem, two stems, three stems and without pruning). The experiment was carried out according to split - split plot in RCBD design with three replicates. Significant differences between the averages were tested in accordance to the polynomial Duncan test. The results showed a significant effect of genotype on length internodes, leaf area and the content of the leaves chlorophyll and the number of days required to flowering of the first flower in $50 \%$ of the plants, while it had no significant effect on plant height and the setting percentage. The magnetically treated water at 3000 gauss had significant effect on plant height, length of the internodes, the content of the leaves chlorophyll and the setting percentage, while no significant effects of water quality on number of branches of the plant and the number of days necessary for the flowering of the first flower in $50 \%$ of the plants. Training methods had no significant effect on plant height, length of internodes, leaf area and the value of chlorophyll in the leaves, while there was significant influence of the training methods on the number of days required to flowering the first flower in 50\% of the plants and the setting percentage.

The tri-interaction among the genotypes and the quality of the water and the methods of training had significant influence, while the genotype NADA plants irrigated with magnetically treated water and training on two stems excelled at setting percentage, while IDEAL plants irrigated with magnetically treated water and left without pruning had the shortest plants. The same genotype (IDEAL) that irrigated with magnetically treated water and trained on three stems had the shortest internodes length and the largest number of side branches. The results show that the genotype Ideal irrigated with natural water and left without pruning gave the highest leaf area. Meanwhile, Ideal plants irrigated with natural water and trained on two stems showed the lowest number of days required for $50 \%$ of flowering.
\end{abstract}

Key words: muskmelon, vegetative growth, flowering, magnetic water. 


\title{
تأثير الماء المعالج مغناطيسياً وطريقة التربية في بعض صفات تاتئ النمو الخضري والزهري

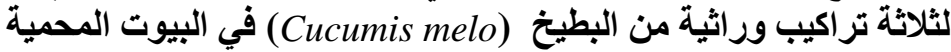

\author{
عزيز مهاي عبد الثمري - رموش حقي اسماعيل
}

قنم البستتة و هندسة الحدائقـ كلية الزر اعة ـ جامعة ديالى ـ العر اق

\begin{abstract}
ملخص دأل

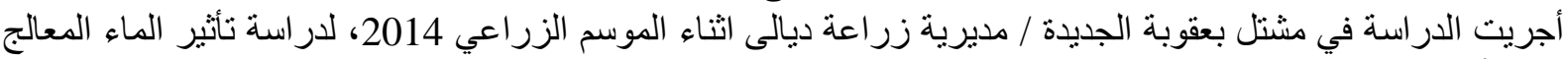

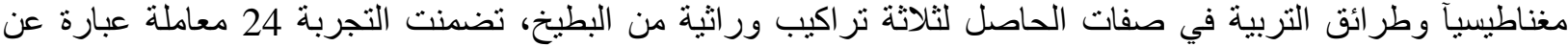

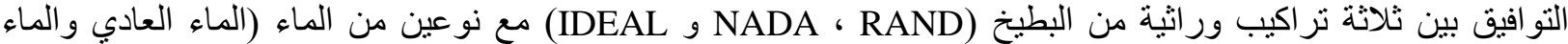

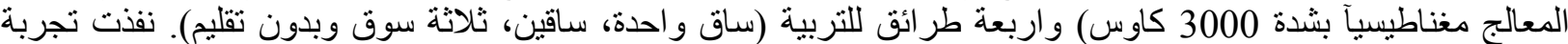

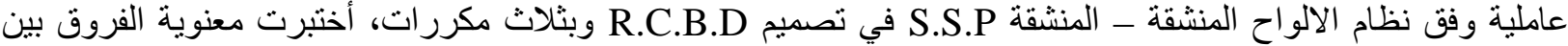

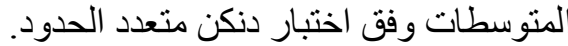

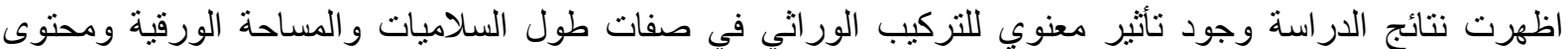

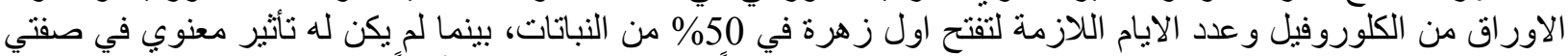

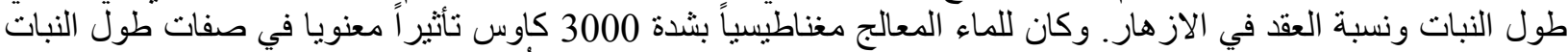

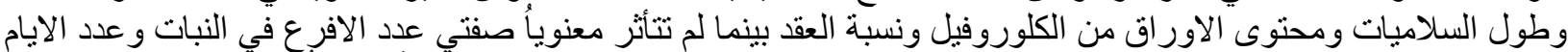

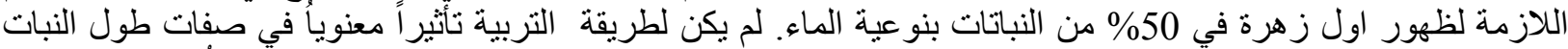

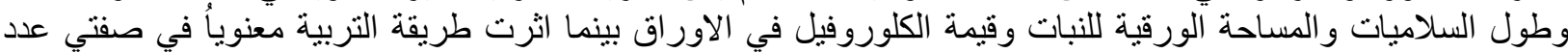

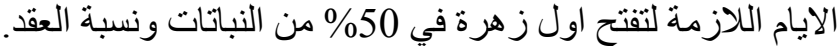

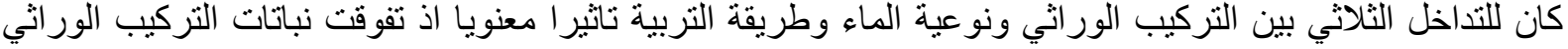

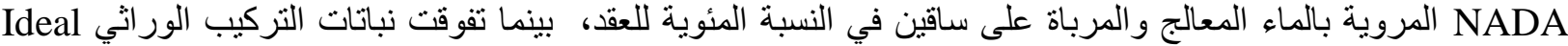

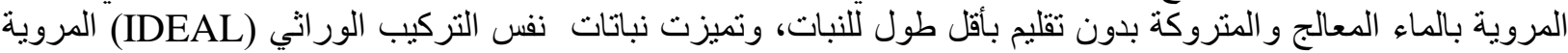

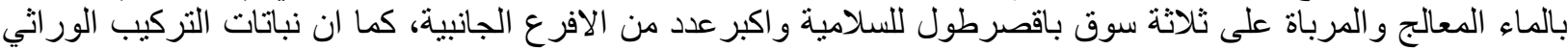

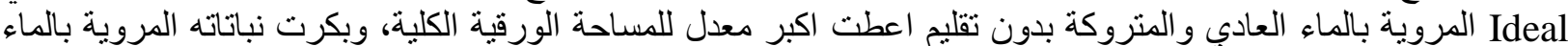

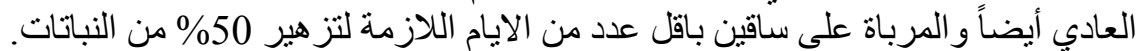

دهون و5.6 غم كربو هيدر ات و و70 ملغم كالسيوم و12 ملغم

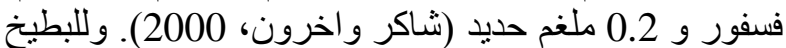

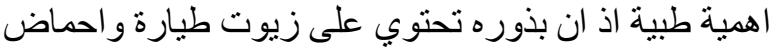

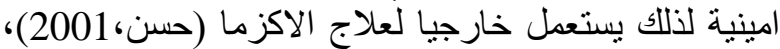

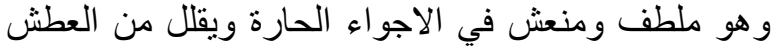

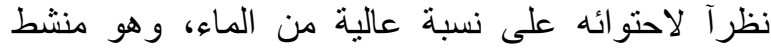

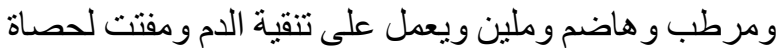
الكلى وتفيد بذوره في تخفيض ضيض ولنط الدم المرتفع وتستعمل

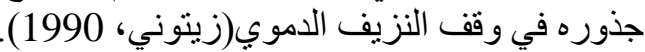

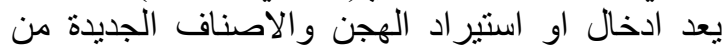
ارخص طرق التربية و التحسين الور اثي والاني ولاسيما في الدول

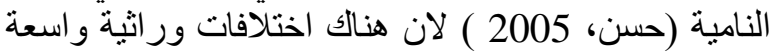

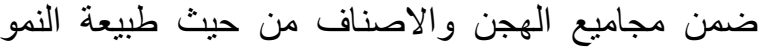
و الانتاج. ذكر كاظم ومحمد (2012) عند در استهم لصنفين الأنين من البطيخ تفوق الصنف المحلي حافظ نفسه على على الهجين

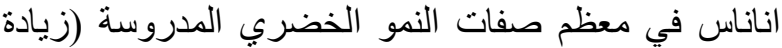

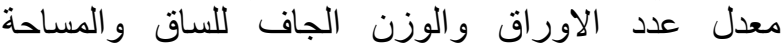

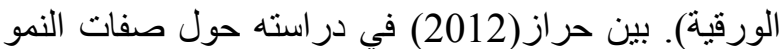

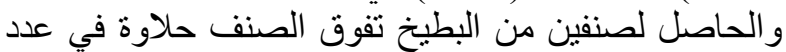

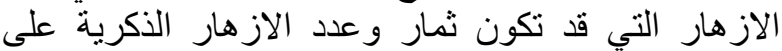

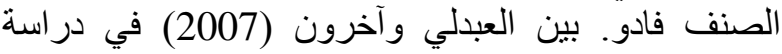
لتحسين صفات النمو الخضري لبعض الاصناف المحلية من

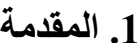

نبات البطيخ (Cucumis melo L.) Muskmelon

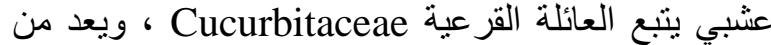
محاصيل الخضر المهمة في العالم، موطنه الاصلي العيل الهند،

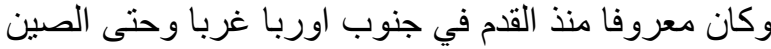

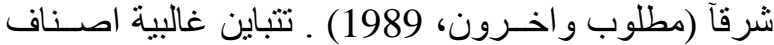

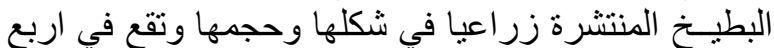

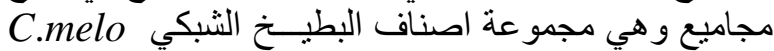
cv. reticulatus C.melo cv.inodorous الكانتلوب C. melo cv. Cantalupensis ، مجمو عة النة اصناف الثمام C. melo cv. aegypticus (حسن، 2001). وللبطيخ اهمية اقتصادية في العالم اذ تجاوزت Yang et المساحة المزروعة منه المليون هكتار في السنة فالسنة (al., 2007)

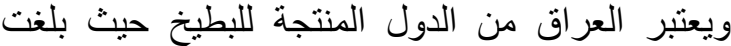
المساحة المزروعة في عام 2012 (66793) دونم بآنتاج اجمالي وصل الى (172.246) طن ولن وبغلة (2.579)

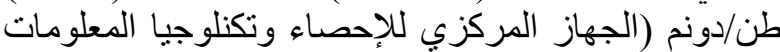

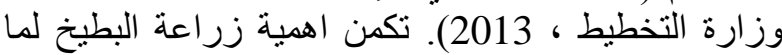

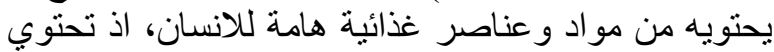
كل 100 غم من ثماره على 0.6 غم بروتينات و0.20.2 غم لمان 
ومعدل طول الساق الرئيس لنبات الطماطة المزروعة في البيوت الزجاجية يقلان معنويا عند التربية على سلى سـاق واحدة

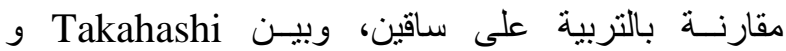
ورود Sasaki

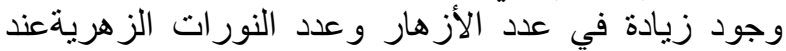

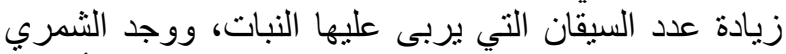

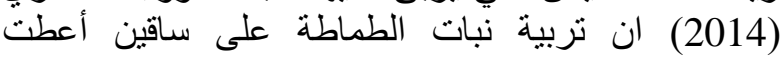
اكبر عدد من النورات الزهرية مقارنة بالنباتات المرباة على الثى

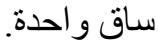
تهذف هذه الدراسة الى اختيار أفضل تركيب ور اثي من

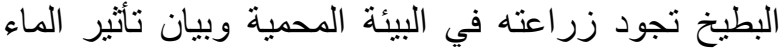

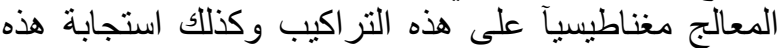
التراكيب لطريقة التربية بما يحقق افضل الترائ نمو خضري

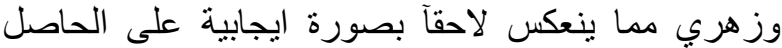

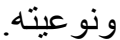

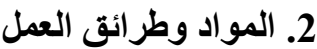

اجريت التجربة الحقلية داخل بيت بلاستيكي فئسة 504 التوبل

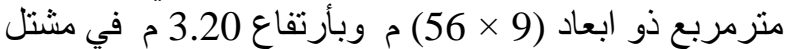

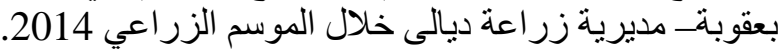

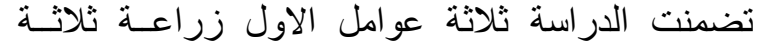

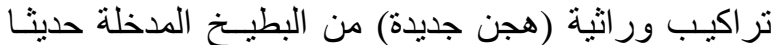

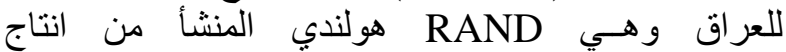
شركة NADA Emmaseed فرنسي المنسأ من انتاج

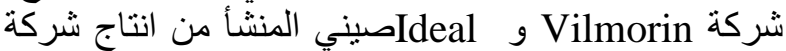
Syngenta

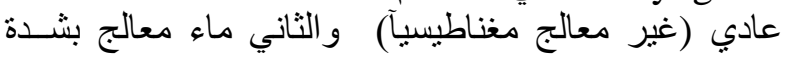

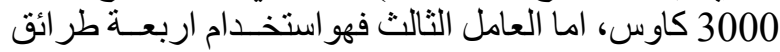
مسن التربية (التربية على سـاق التهاق واحدة، وعلى ساقين، و على ثلاثة سيقان وبدون تقليم ).

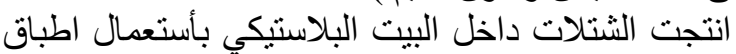

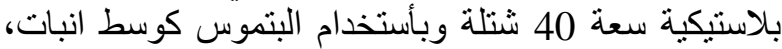
زر عت البذور بتاريخ 2014/1/7 وبو اقع بذرة والتهات واحدة في كل

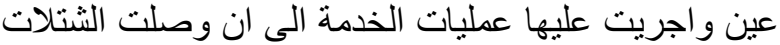

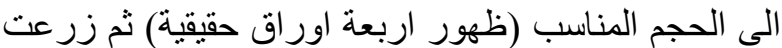

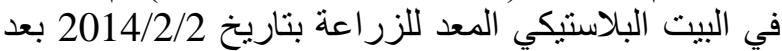

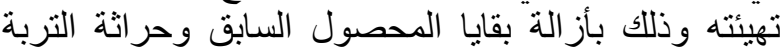
لاكثر من مرة وتتعيمها وتسويتها جيدا ثم اجريت التيان عليها

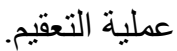
زرعت الثتلات على اربعة مصاطب بعرض 80 سم سم وبخطين زراعة لكل مصطبة وبمسافة 40 سم بين نئ نبات واخر. بلغ طول الخط الو احد 3م لكل وحدة تجريبية يحتوي

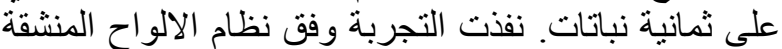
- المنشقة Split- Split plot design ضمن تصميم القطاعات العشوائية الكاملة RCBD، اذ وضعت الهجن في

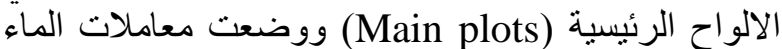

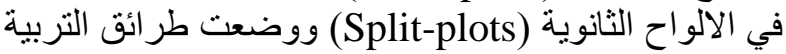

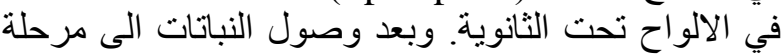

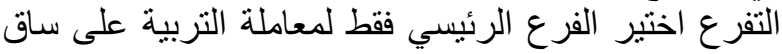
واحدة وفر عين (الرئيسي وفرع الريسي جانبي) لطريقة التربية على للى على
البطيخ وهي حافظ نفسه و الاسماعيلي و الأناناس ان هناك الكي

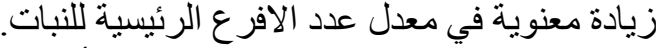

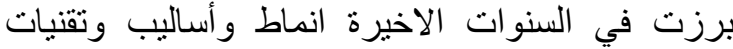

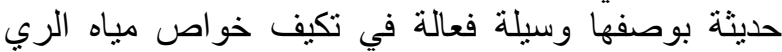

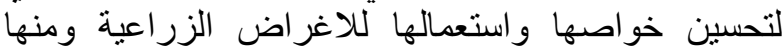
التقنية المغناطيسية (الجوذري، 2006 ). إن استعمال التقنية لزية التراعية

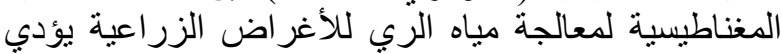

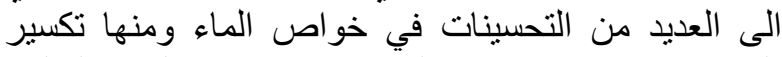

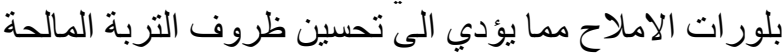

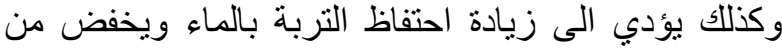

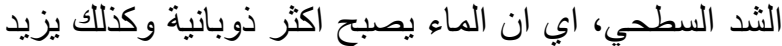

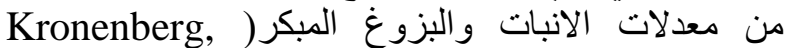
2005). بين العبيدي وآخرون (2012) ان النيزون استخدام المياه

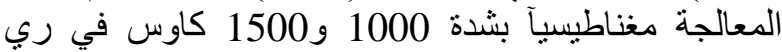
نبات الخيار صنف سارة المزروع في البيت البلاستيكي ادى في

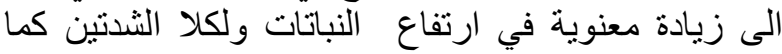

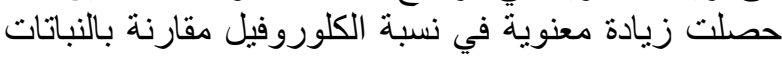

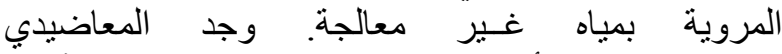
واخرون (2009) أن استعمال المياه المعالجة مغناطيسياً اثر المرئ

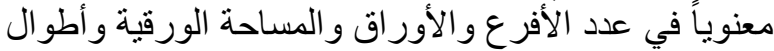

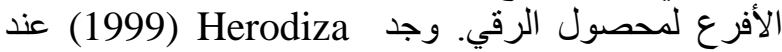

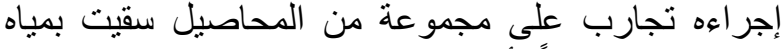

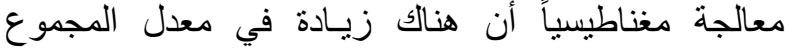

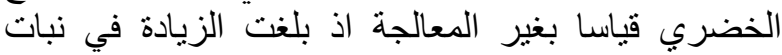

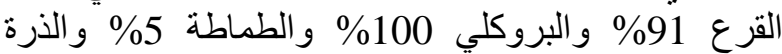
40\% و البصل \%4\% \% و وجدت حسن و آخرون (2011) عند

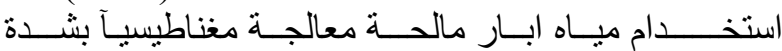

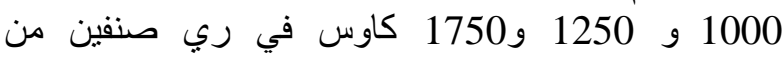
الطماطة تفوق و اضح للنباتات المروية بالماء المعالج بشدة

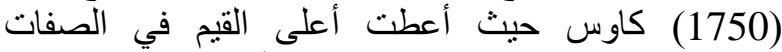
الخضرية مثل ارتفاع النبات وعدد الأفرع وطول الوبل الورقة الوقة

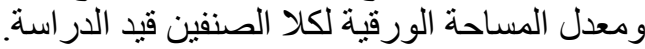

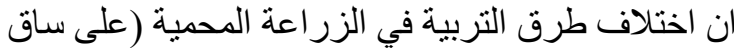

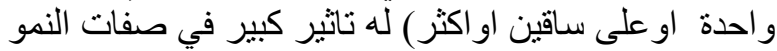

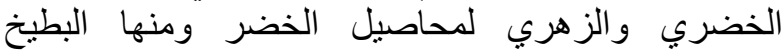
(المؤمن، 1991)، وتعد طريقة التربية من العوامل المبل المؤثرة

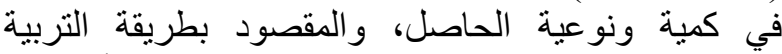

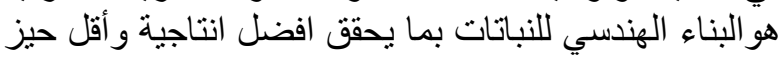

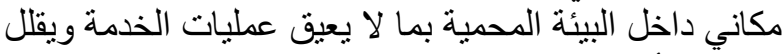

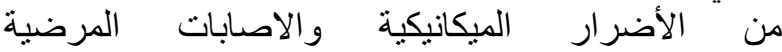
(العبدلي، 2007). ان تربية وتقليم النباتات تعطيها القدرة القابة

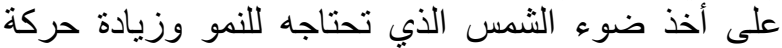

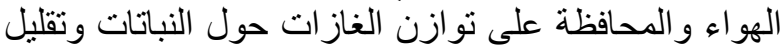
خطر الإصابة بالفطريات والحشر اتش ات (19) (Guo et al.,1991) بين الحربي وآخرون (1996) تفوف نباتات الخيار المربات الحئي

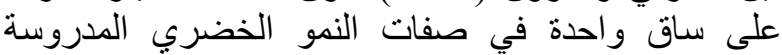
مقارنة مع النباتات المرباة على ساقين ووجد سعود الني (2013)

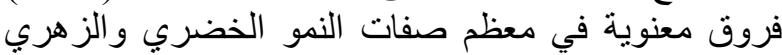

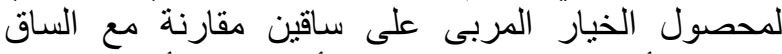
الواحدة، أوضح كاظم (1986) أنّ عدد الأوراق الكلين 
توضح نتائج الجدول (1) أن التراكيب الور اثية لم تؤثر

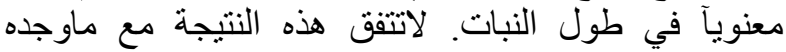

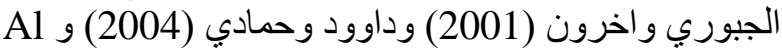

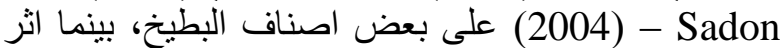

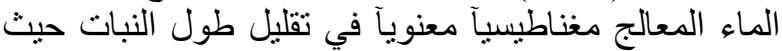

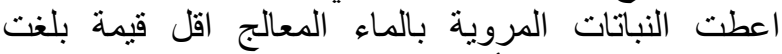

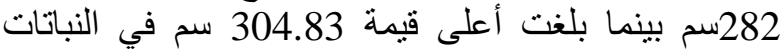

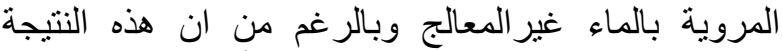
لايتفق مع ما وجده الجوذري (2006) فأن قلة طول الئ النداء النبات

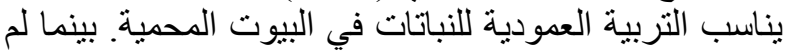
تؤثر طر ائق التربية معنوياً في قيمة طول النبات النبات.

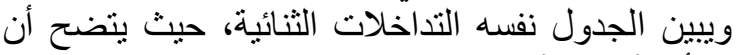

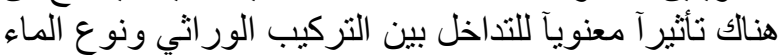

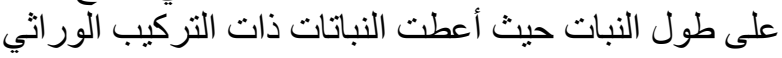
IDEAL بلغت 241.58 سم بينما اعطت النباتات ذات الترات التركيب الور اثي NADA والمروي بالماء غير المعالج اعلى طول

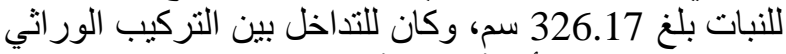

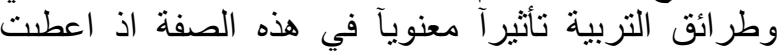

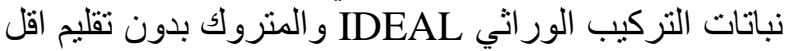

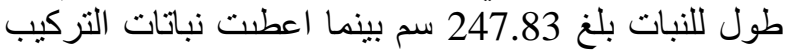

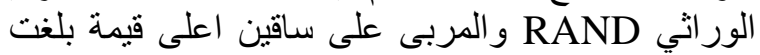

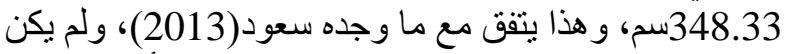
للتناخل بين طريقة التربية ونوعية الماء اي تأثير معنوي ولميكي ل ل لهذه الصفة.

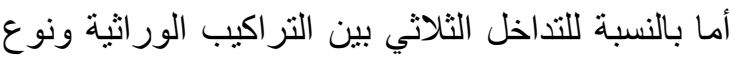

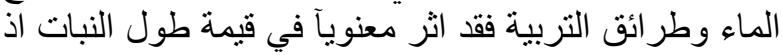

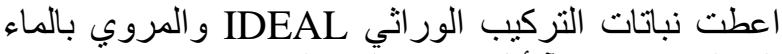

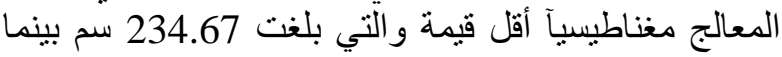

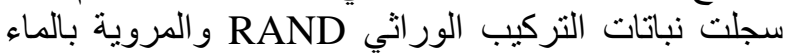
غير المعالج والمرباة على ساقين أعلى قيمة بلغت

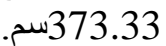

ربما يرجع سبب انخفاض قيمة طول النبات الى قصر

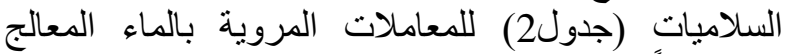

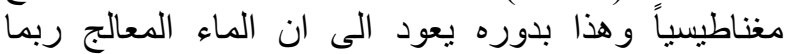
يخفض من تأثير قلة الاضاءة التي تسبب استطالة السلاميات ومن ثم استطالة النبات.

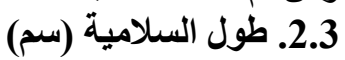
تشير نتائج الجدول (2 ) التئه ) الى وجود فروق معنوية بين

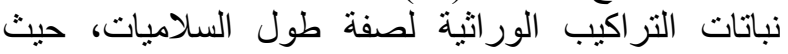

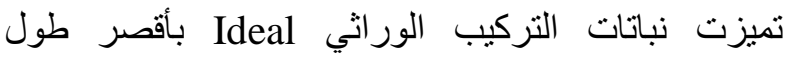

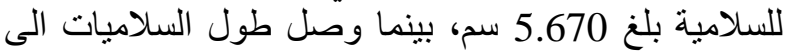

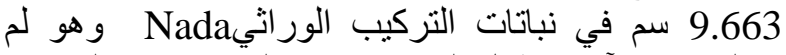
يختلف معنوياً عن طول السلاميات في النباتات ذات اتلات التركيب

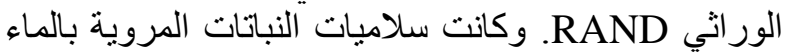

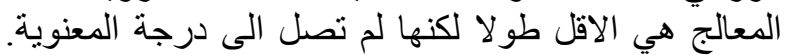

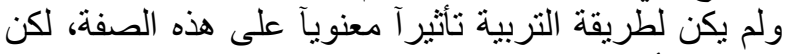

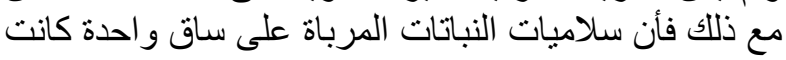
هي الاقل طو لا.
ساقين وثلاثة فروع (الرئيسي وفر عين جانبيين) للتربية على التى

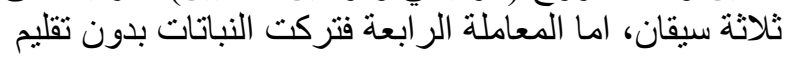

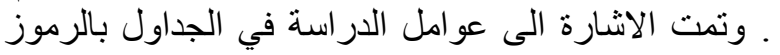

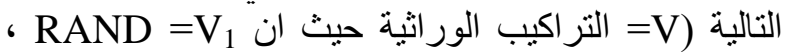
NADA =V حيث ان Sم= ماء غير معالج و 3000 كاوس) و ( طر ائق التربية حيث ان مان

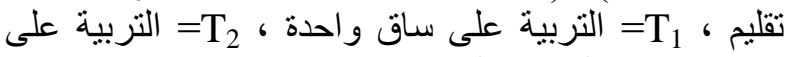

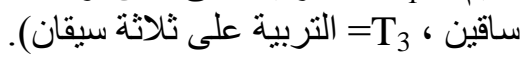

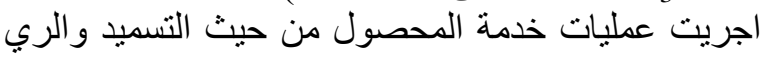
وتسليق النباتات حسب الحاجة واخذت العينات من خمسة نباتات عشوائية من كل وحدة تجريبية ودرست الصفات التفات

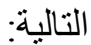
1- منوسط طول النبات (سم): نم قياس طول النبات في نهاية

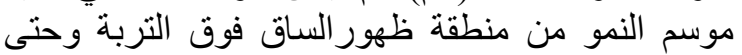

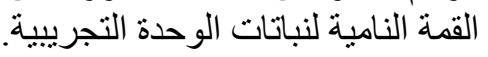

2- طول السلامية (سم): نم قياس طول طلات السربية السلامية في نهاية

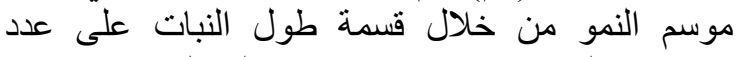
سلامياته ولخمسة نباتات ثم استخر جاند المعدل.

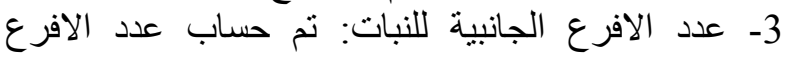
الجانبية المتكونة على الساق الرئيسية في نهاية موسم الأب

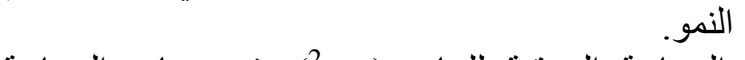
4- المساحة الورقية للنبات (دسم): تم حساب المساحة الورقية بوساطة جهاز (Area meter Am 300) حيث قيست المساحة الورقية لخمسة اوراق مختلفة

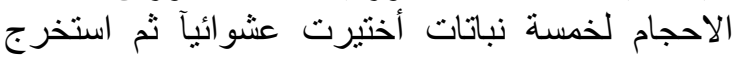
معدل مساحة الورقة الواحدة. ثم استخرجت المثن المساحة

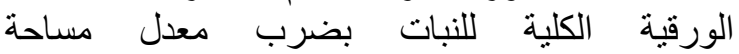

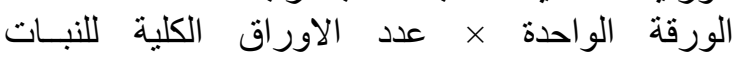
.Wallace et al ., 2000) 5- محتوى الأوراق من الكلوروفيل (SPAD): تم حسابها

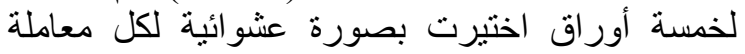

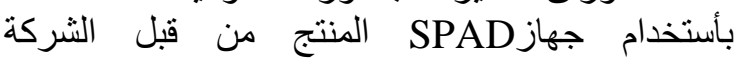
Minolta 6- عدد الأيام لحين تفتح أول زهرة في 50\% من من النباتات

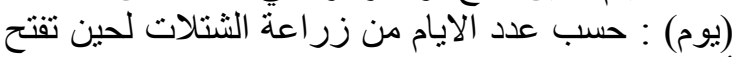

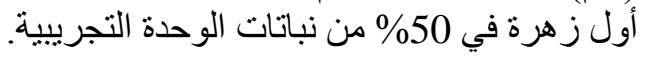

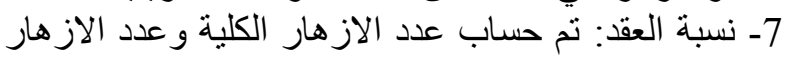

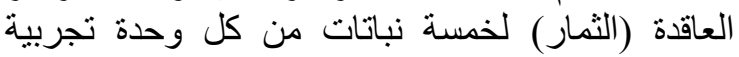
وحسبت نسبة العقد وفق المعادلة الاتية: نسبة العقد = (عدد الازهار العاقدة / عدد الاز هار الكلية ) × 100 تم تحليل النتائج احصائيا وحسب التصميم المستخدم في بائ

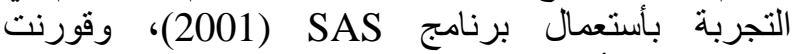
المتوسطات بأختبار دنكن متعدد الحدود (الراوي وخلف) الله، 2000).

\section{3. النتائج والمناقشة}

1.3. طول النبات (سم) 
جدول (1): تأثير التركيب الوراثي والماء المعالج مغناطيسياً وطرائق التربية وتداخلاتها في طول النبات (سم).

\begin{tabular}{|c|c|c|c|c|c|c|}
\hline \multirow[t]{2}{*}{ التداخل V×W } & \multicolumn{4}{|c|}{ طرائق التربية } & \multirow[t]{2}{*}{ نوعية الماء } & \multirow{2}{*}{ للوراثية } \\
\hline & \multicolumn{2}{|r|}{ T2 } & T1 & T0 & & \\
\hline 320.83 & 323.33 & \multirow{2}{*}{$\begin{array}{c}373.33 \\
\text { a }\end{array}$} & 310.00 & 267.67 & W0 & \multirow{4}{*}{ V1 } \\
\hline $\mathbf{a}$ & a-d & & a-d & Bed & & \\
\hline 311.42 & 300.67 & \multirow{2}{*}{$\begin{array}{c}323.33 \\
\text { a - d }\end{array}$} & 326.67 & 295.00 & W1 & \\
\hline $\mathbf{a}$ & a-d & & abc & a-d & & \\
\hline 326.17 & 329.33 & \multirow{2}{*}{$\begin{array}{c}309.33 \\
\text { a-d }\end{array}$} & 343.33 & 322.67 & W0 & \multirow[t]{4}{*}{$\mathbf{V 2}$} \\
\hline a & abc & & ab & a-d & & \\
\hline 293.00 & 311.00 & \multirow{2}{*}{$\begin{array}{c}291.00 \\
\text { a-d }\end{array}$} & 271.67 & 298.33 & W1 & \\
\hline $\mathbf{a b}$ & a-d & & bcd & a-d & & \\
\hline 267.50 & 258.33 & \multirow{2}{*}{$\begin{array}{c}271.67 \\
\text { bed }\end{array}$} & 279.00 & 261.00 & Wo & \multirow{4}{*}{$\mathbf{V 3}$} \\
\hline bc & bed & & bed & Bcd & & \\
\hline 241.58 & 240.00 & \multirow{2}{*}{$\begin{array}{c}247.33 \\
\text { cd }\end{array}$} & 244.33 & 234.67 & W1 & \\
\hline c & cd & & cd & D & & \\
\hline & 293.78 & \multirow{2}{*}{$\begin{array}{c}302.67 \\
\text { A }\end{array}$} & 295.83 & 281.39 & \multirow{2}{*}{\multicolumn{2}{|c|}{ متوسطات طرائق التربية }} \\
\hline & $\mathbf{A}$ & & $\mathbf{A}$ & A & & \\
\hline \multicolumn{7}{|c|}{ التداخل الثنائي بين التراكيب الوراثية وطرائق التربية } \\
\hline \multirow{2}{*}{ متوسطات التراكيب } & \multicolumn{4}{|c|}{ طرائق التربية } & \multirow{2}{*}{\multicolumn{2}{|c|}{ التراكيب الوراثية }} \\
\hline & T3 & T2 & T1 & T0 & & \\
\hline 316.13 & 312.00 & 348.33 & 318.44 & 285.83 & \multirow{2}{*}{\multicolumn{2}{|c|}{ V1 }} \\
\hline $\mathbf{A}$ & $\mathbf{A b}$ & $\mathbf{a}$ & ab & Bc & & \\
\hline 309.58 & & 300.17 & 307.50 & 310.50 & \multirow{2}{*}{\multicolumn{2}{|c|}{$\mathbf{V 2}$}} \\
\hline $\mathbf{A}$ & $\mathbf{A b}$ & abc & abc & Ab & & \\
\hline 254.54 & \multirow{2}{*}{$\begin{array}{c}249.17 \\
\text { c }\end{array}$} & 259.50 & 261.67 & 247.83 & \multirow{2}{*}{\multicolumn{2}{|c|}{$\mathbf{V 3}$}} \\
\hline $\mathbf{A}$ & & bc & bc & $\mathbf{C}$ & & \\
\hline \multicolumn{7}{|c|}{ التداخل الثنائي بين نوعية الماء وطرائق التربية } \\
\hline متوسطات نوعية & \multicolumn{4}{|c|}{ طر ائق التربية } & & نوعيا \\
\hline الماء & T3 & T2 & T1 & T0 & & \\
\hline 304.833 & 303.67 & 318.11 & 310.78 & 286.78 & & \\
\hline $\mathbf{A}$ & $\mathbf{a}$ & $\mathbf{a}$ & $\mathbf{a}$ & A & & \\
\hline 282.00 & 283.89 & 287.22 & 280.89 & 276.00 & & \\
\hline B & $\mathbf{a}$ & $\mathbf{a}$ & $\mathbf{a}$ & A & & \\
\hline
\end{tabular}

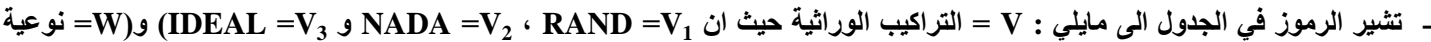
الماء حيث ان

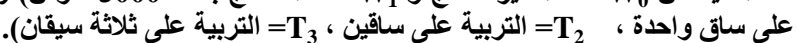
ــ القيم المتبوعة بنفس الحرف لا يوجد بينها فروق معنوية طبقا لاختبار دنكن متعدد الحدود. 
جدول (2) : تأثير التركيب الوراثي والماء المعالج مغتاطيسيا وطرائق التربية وتداخلاتها في طول السلامية (سم).

\begin{tabular}{|c|c|c|c|c|c|c|}
\hline \multirow[t]{2}{*}{ V×W } & \multicolumn{4}{|c|}{ طرائق التربية } & \multirow[t]{2}{*}{ نوعية الماء } & \multirow{2}{*}{ التراثيب } \\
\hline & T3 & $\mathbf{T 2}$ & T1 & T0 & & \\
\hline 8.705 & 8.184 & \multirow{2}{*}{$\begin{array}{l}9.628 \\
a-d\end{array}$} & 7.288 & 9.722 & W0 & \multirow{4}{*}{ V1 } \\
\hline $\mathbf{a}$ & $\mathbf{a}-\mathbf{f}$ & & $\mathbf{d}-\mathbf{h}$ & $\mathbf{a}-\mathbf{d}$ & & \\
\hline 8.347 & 7.947 & \multirow{2}{*}{$\begin{array}{l}7.381 \\
c-h\end{array}$} & 7.497 & 10.564 & W1 & \\
\hline $\mathbf{a}$ & $\mathbf{b}-\mathbf{g}$ & & $\mathbf{c}-\mathbf{h}$ & $\mathbf{a b}$ & & \\
\hline 10.184 & 9.989 & \multirow{2}{*}{$\begin{array}{c}10.368 \\
\text { Ab }\end{array}$} & 10.676 & 9.704 & W0 & \multirow{4}{*}{$\mathbf{V 2}$} \\
\hline $\mathbf{a}$ & abc & & $\mathbf{a}$ & $\mathbf{a}-\mathbf{d}$ & & \\
\hline 9.141 & 9.437 & \multirow{2}{*}{$\begin{array}{l}9.237 \\
a-d\end{array}$} & 9.495 & 8.398 & W1 & \\
\hline $\mathbf{a}$ & $\mathbf{a}-\mathbf{d}$ & & $\mathbf{a}-\mathbf{d}$ & $\mathbf{a}-\mathbf{e}$ & & \\
\hline 5.789 & 6.104 & \multirow{2}{*}{$\begin{array}{c}6.040 \\
f-i\end{array}$} & 5.296 & 5.716 & W0 & \multirow{4}{*}{$\mathbf{V 3}$} \\
\hline b & $e-\mathbf{i}$ & & hi & $\mathbf{f}-\mathbf{i}$ & & \\
\hline 5.552 & 4.698 & \multirow{2}{*}{$\begin{array}{c}5.530 \\
\text { Ghi }\end{array}$} & 5.357 & 6.624 & W1 & \\
\hline \multirow[t]{3}{*}{ b } & i & & ghi & $e-\mathbf{i}$ & & \\
\hline & 7.726 & \multirow{2}{*}{$\begin{array}{c}8.030 \\
\text { A }\end{array}$} & 7.601 & 8.454 & \multirow{2}{*}{\multicolumn{2}{|c|}{ متوسطات طرائق التربية }} \\
\hline & $\mathbf{A}$ & & $\mathbf{A}$ & $\mathbf{A}$ & & \\
\hline \multicolumn{7}{|c|}{ التداخل الثنائي بين التراكيب الوراثية وطرائق التربية } \\
\hline \multirow{2}{*}{ متوسطات التر اكيب } & \multicolumn{4}{|c|}{ طرائق التربية } & \multirow{2}{*}{\multicolumn{2}{|c|}{ التراكيب الوراثية }} \\
\hline & T3 & $\mathbf{T 2}$ & T1 & T0 & & \\
\hline 8.526 & 8.065 & 8.504 & 7.392 & 10.143 & \multicolumn{2}{|c|}{ V1 } \\
\hline B & bc & abc & cd & $\mathbf{a}$ & & \\
\hline 9.663 & 9.713 & 9.802 & 10.085 & 9.051 & & 2 \\
\hline $\mathbf{A}$ & $\mathbf{a b}$ & $\mathbf{a b}$ & $\mathbf{a}$ & abc & & \\
\hline 5.670 & 5.401 & 5.785 & 5.326 & 6.170 & & 3 \\
\hline C & $\mathbf{e}$ & de & $\mathbf{e}$ & de & & \\
\hline & & وطرائق التربية & ين نوعية الا & التداخل ال & & \\
\hline 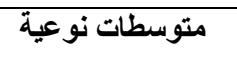 & & تربية & 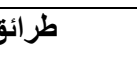 & & & نوعيةً \\
\hline الماء & T3 & $\mathbf{T} 2$ & T1 & T0 & & \\
\hline 8.226 & 8.092 & 8.678 & 7.753 & 8.380 & & $\mathbf{0}$ \\
\hline $\mathbf{A}$ & $\mathbf{a}$ & $\mathbf{a}$ & $\mathbf{a}$ & $\mathbf{a}$ & & \\
\hline 7.680 & 7.360 & 7.382 & 7.449 & 8.528 & & 1 \\
\hline $\mathbf{A}$ & $\mathbf{a}$ & $\mathbf{a}$ & $\mathbf{a}$ & $\mathbf{a}$ & & \\
\hline
\end{tabular}

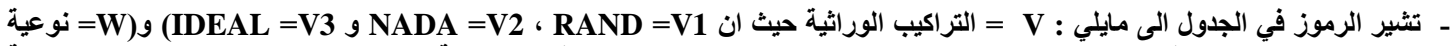

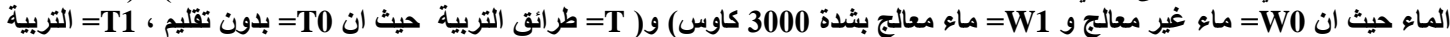

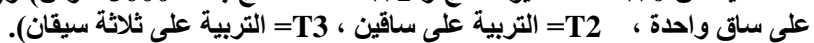
ـ القيم المتبوعة بنفس الحرف لا يوجد بينها فروق معنوية طبقا لاختبار دنكن متعدد الحدود. 
التركيب الوراثي NADA و المربى على ساق واحدة اقل

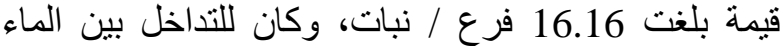

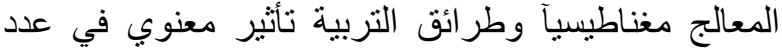
الافرع الجانبية للنبات حيث اعطت النيات النباتات المروية باتية بالماء

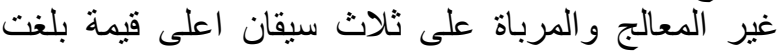

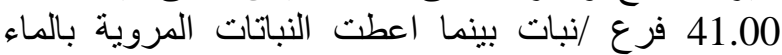

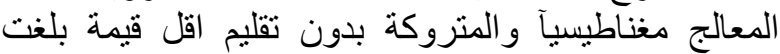
20.55

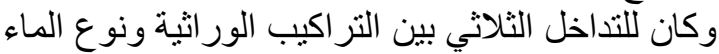

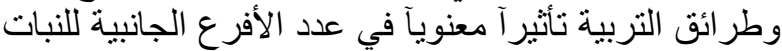

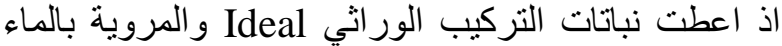

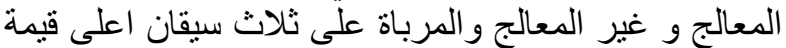

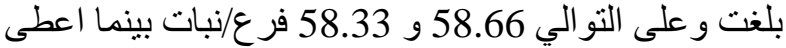
التركيب الور اثي NADA و المروي بالماء غلي غير فير المعالج

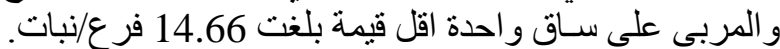
4.3. المساحة الورقية للنبات (دسم²)

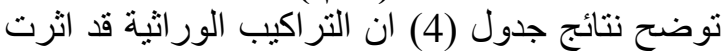

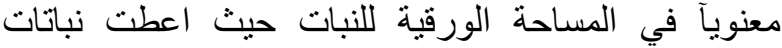
التركيب الوراثي Ideal اعلى قيمة للمساحة الورقية الكلية

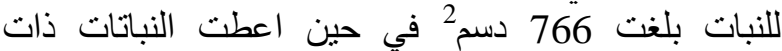

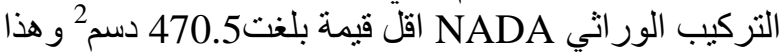
يتفق مع ماوجدته الدوري (2010) بينما لاتتفق هذه النتيجة

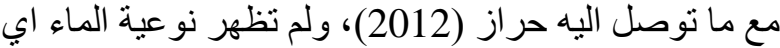

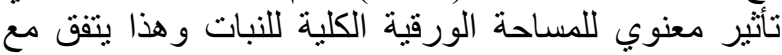

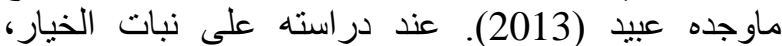

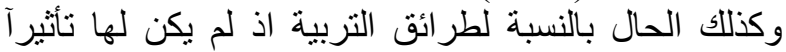

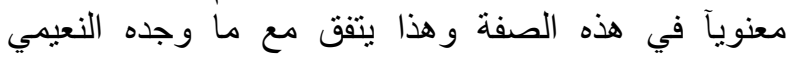

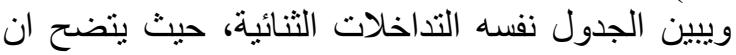
هناك فرق معنوي للتداخل بين التركيب الوراني التئ والتياء

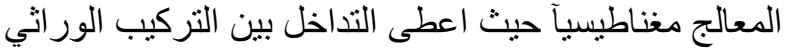

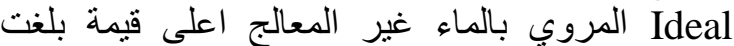

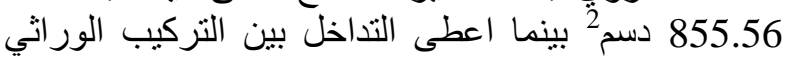

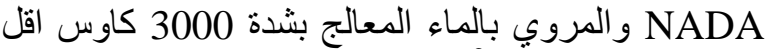

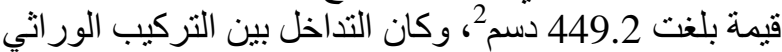

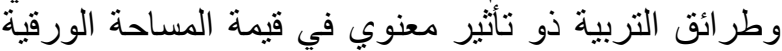

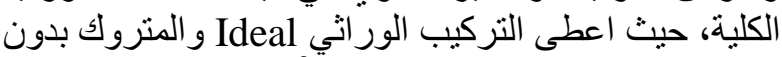
تقليم اعلى قيمة بلغت واعلى التركيب 804.94 دسمّ2، بينما اعطى التركيب

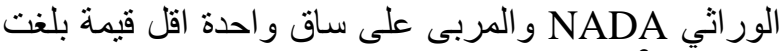

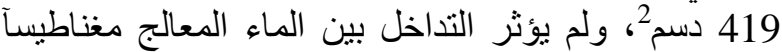
وطر ائق التربية معنوياً في قيمة المساحة الورقية لتئ الكية الكلية.

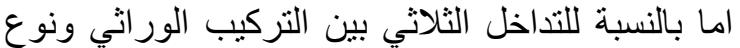

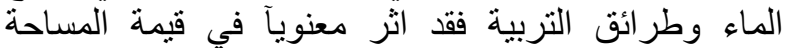

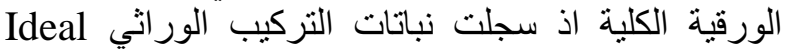

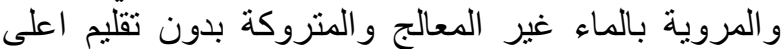

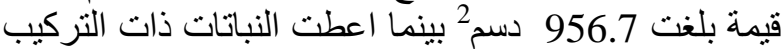

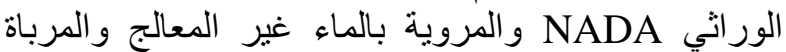
على سأق واحدة اقل مساحة ورقية بلغت بلئ 352.5 دسم2.
وتوضح النتائج بأن هناك تأثثرآ معنوياً للتداخل بين

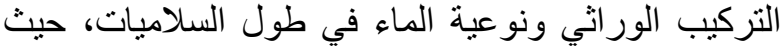
تميزت نباتات التركيب الور اثي Ideal سواء المراء المروية منهات بالماء المعالج او غير المعالج بأقصر طول للس للسلاميات فبلغ

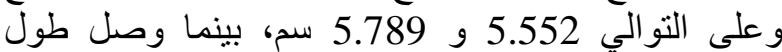
السلاميات الى الى 10.184 سم في نباتات التركيب الور اثني

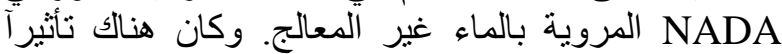

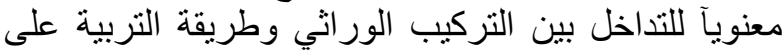

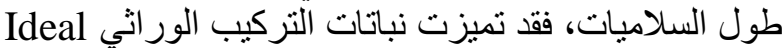

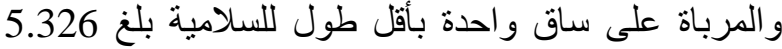

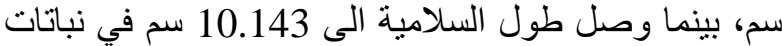
التركيب الور اثي RAND غير المقلمة. ولم يكن للتنداخل بين نوعية الماء وطريقة التربية تأثيرآ معنويآ على طول

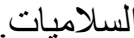

وتثير المعلومات الواردة في الجدول 2 بأن هناك تأثنيراً معنوياً للتداخل الثلاثي بين التراكي أكيب الور اثية ونية ونوعية الماء

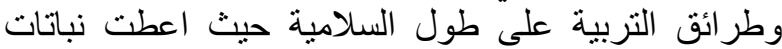

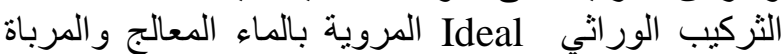
على ثناثة سوق اقل طول للسلامية بلغ 4.698 سم، بينما اعطت نباتات التركيب الوراثي NADA المروية بالماء غير المعالج والمرباة على ساق واحدة اعلى طول للسلامية بلغ 10.676 سم. 3.3. عدد الأفرع الجانبية للنبات (فرع / نبات)

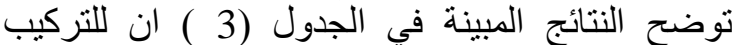

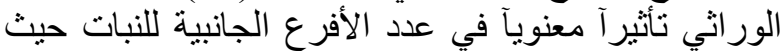

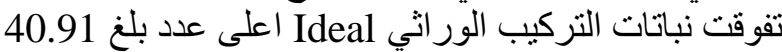

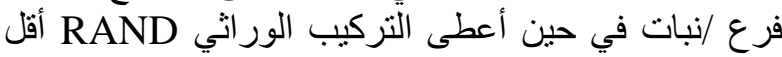

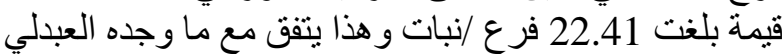
(2007) وحسين (2002) وحراز (2012) ويعود السبب النيب الى ان عدد الافرع الجانبية يتحكم في توريثها عدد من

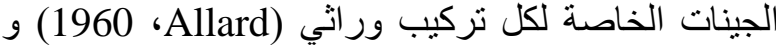
Edwards و Lower)

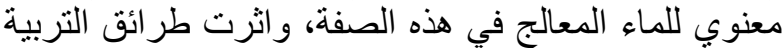

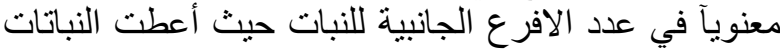
المرباة على ثلاث سيقان أعلى قيمة بلغت بينما بلغت أقل قيمة 21.66 فر ع /نبات في النبات النباتات المنروكة بدون تقليم.

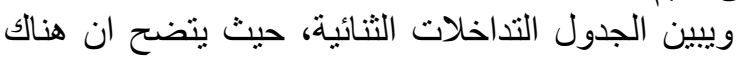

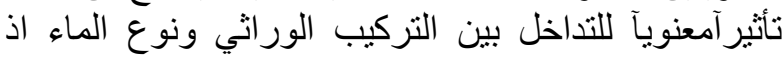
تميزت نباتات التركيب الور اثي Ideal سو اء المروية المئية منها

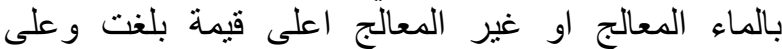
الترتيب 44 و 37.83 فرع / نبات بينمات الوريا اعطى التركيب الوراثي RAND و المروي بالماء غير الثير المعالج اقل قيمة بلغت 22.083 فرع / نبات وقد يكون السبب الت الن النباتات

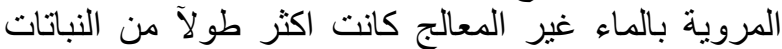

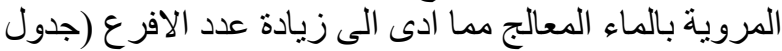

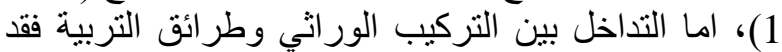

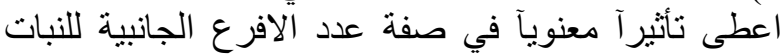

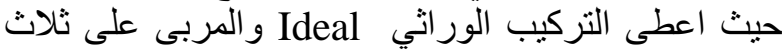
سيقان اعلى قيمة بلغت 58.50 فرع /نبات بينما اعطى 
جدول (3): تأثثر التركيب الوراثي والماء المعالج مغناطيسياً وطرائق التربية وتداخلاتها في عدد الافرع الجانبية

\begin{tabular}{|c|c|c|c|c|c|c|}
\hline & & & & & ت فرع/ نبات & \\
\hline التداخل $\mathbf{V} \times \mathbf{W}$ & & ق التربية & & & نوعية الماء & التراكيب \\
\hline & T3 & $\mathbf{T 2}$ & T1 & T0 & & الوراثية \\
\hline 22.083 & 26.333 & 26.667 & 18.667 & 16.667 & W0 & \\
\hline b & c-g & c-g & d-g & fg & & \\
\hline 22.750 & 32.00 & 24.333 & 17.00 & 17.667 & W1 & \\
\hline b & c-f & c-g & fg & efg & & \\
\hline 25.583 & 38.333 & 31.00 & 14.667 & 18.333 & W0 & \\
\hline b & b-c & c-f & $\mathbf{g}$ & d-g & & \\
\hline 24.417 & 31.667 & 25.00 & 17.667 & 23.333 & W1 & 2 \\
\hline b & c-f & c-g & efg & c-g & & \\
\hline 44.00 & 58.333 & 50.667 & 33.667 & 33.333 & W0 & \\
\hline $\mathbf{a}$ & $\mathbf{a}$ & bc & cd & cde & & \\
\hline 37.833 & 58.667 & 38.667 & 33.333 & 20.667 & W1 & \\
\hline $\mathbf{a}$ & $\mathbf{a}$ & bc & cde & d-g & & \\
\hline & 40.889 & 32.722 & 22.500 & 21.667 & ائق التربية & متوسطات \\
\hline & $\mathbf{A}$ & B & $\mathbf{C}$ & $\mathbf{C}$ & & \\
\hline & & الثية وطرائق ال & ن التراكيب الر & التـاخل الثنائي & & \\
\hline متوسطات التراكيب & & لتربية & طر ائق & & ل يراثية & 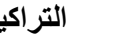 \\
\hline الوزايكי & T3 & $\mathbf{T 2}$ & T1 & T0 & & \\
\hline 22.417 & 29.167 & 25.500 & 17.833 & 17.167 & & \\
\hline B & cd & c-g & efg & fg & & \\
\hline 25.00 & 35.00 & 28.00 & 16.167 & 20.833 & & \\
\hline B & c & cde & $\mathbf{g}$ & d-g & & \\
\hline 40.917 & $\mathbf{5 8 . 5 0 0}$ & 44.667 & 33.500 & 27.00 & & \\
\hline $\mathbf{A}$ & $\mathbf{a}$ & b & c & c-f & & \\
\hline & & ؛ وطرائق التربياً & بين نوعية اله & التـاخل الثنا & & \\
\hline متوسطات نوعية & & لتربية & طر ائق & & & نوء \\
\hline الماء & T3 & $\mathbf{T 2}$ & T1 & T0 & & \\
\hline 30.556 & 41.00 & 36.111 & 22.333 & 22.778 & & \\
\hline $\mathbf{A}$ & $\mathbf{a}$ & ab & cd & cd & & \\
\hline 28.333 & 40.778 & 29.333 & 22.667 & 20.556 & & \\
\hline $\mathbf{A}$ & $\mathbf{a}$ & bc & cd & d & & \\
\hline
\end{tabular}

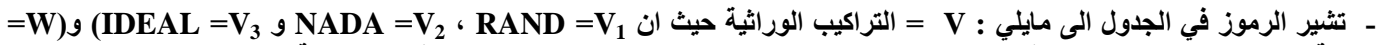

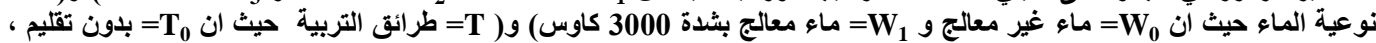
الت T T ـ القيم المتبوعة بنفس الحرف لا يوجد بينها فروق معنوية طبقا لاختبار دنكن متعدد الحدود. 
جدول (4) : تأثير التركيب الوراثي والماء المعالج مغناطيسياً وطرائق التربية وتداخلاتها في المساحة الورقية

\begin{tabular}{|c|c|c|c|c|c|c|}
\hline \multirow[t]{2}{*}{ V×W } & \multicolumn{4}{|c|}{ طر ائق التربية } & \multirow[t]{2}{*}{ نوعية الماء } & \multirow{2}{*}{ التوراثية } \\
\hline & T3 & $\mathbf{T 2}$ & T1 & T0 & & \\
\hline 483.17 & 447.4 & \multirow{2}{*}{$\begin{array}{c}488.3 \\
\text { cde }\end{array}$} & 504.0 & 492.9 & Wo & \multirow[t]{4}{*}{ V1 } \\
\hline c & cde & & cde & cde & & \\
\hline 516.35 & 434.7 & \multirow{2}{*}{$\begin{array}{c}440.6 \\
\text { cde }\end{array}$} & 582.7 & 607.4 & W1 & \\
\hline c & cde & & b - e & $a-e$ & & \\
\hline 491.78 & 523.7 & \multirow{2}{*}{$\begin{array}{l}593.5 \\
\text { b - e }\end{array}$} & 352.5 & 497.5 & W0 & \multirow{4}{*}{$\mathbf{V 2}$} \\
\hline c & cde & & e & cde & & \\
\hline 449.20 & 508.1 & \multirow{2}{*}{$\begin{array}{c}432.4 \\
\text { cde }\end{array}$} & 485.5 & 370.8 & W1 & \\
\hline c & cde & & cde & de & & \\
\hline 855.56 & 756.2 & \multirow{2}{*}{$\begin{array}{c}909.2 \\
\text { ab }\end{array}$} & 800.2 & 956.7 & W0 & \multirow[t]{4}{*}{ V3 } \\
\hline $\mathbf{a}$ & abc & & abc & $\mathbf{a}$ & & \\
\hline 676.43 & 739.3 & \multirow{2}{*}{$\begin{array}{l}679.7 \\
a-e\end{array}$} & 633.5 & 653.2 & W1 & \\
\hline \multirow{3}{*}{ b } & $\mathbf{a}-\mathbf{d}$ & & $\mathbf{a}-\mathbf{e}$ & a - e & & \\
\hline & 568.25 & \multirow{2}{*}{$\begin{array}{c}590.62 \\
\text { A }\end{array}$} & 559.72 & 596.41 & \multirow{2}{*}{\multicolumn{2}{|c|}{ متوسطات طرائق التربية }} \\
\hline & $\mathbf{A}$ & & $\mathbf{A}$ & $\mathbf{A}$ & & \\
\hline \multicolumn{7}{|c|}{ التداخل الثنائي بين التراكيب الوراثية وطرائق التربية } \\
\hline \multirow{2}{*}{ متوسطات التراكيب } & \multicolumn{4}{|c|}{ طرائق التربية } & \multirow{2}{*}{\multicolumn{2}{|c|}{ التراكيب الوراثية }} \\
\hline & T3 & T2 & T1 & T0 & & \\
\hline 499.8 & 441.1 & 464.5 & $\begin{array}{l}543.4 \\
\end{array}$ & 550.1 & \multirow{2}{*}{\multicolumn{2}{|c|}{ V1 }} \\
\hline $\mathbf{A B}$ & c & c & bc & bc & & \\
\hline 470.5 & 515.9 & 512.9 & 419.0 & 434.2 & \multirow{2}{*}{\multicolumn{2}{|c|}{ V2 }} \\
\hline B & bc & bc & c & c & & \\
\hline 766.0 & 747.8 & 794.5 & 716.8 & 804.9 & \multirow{2}{*}{\multicolumn{2}{|c|}{ V3 }} \\
\hline $\mathbf{A}$ & $\mathbf{a b}$ & $\mathbf{a}$ & $\mathbf{a b}$ & $\mathbf{a}$ & & \\
\hline \multicolumn{7}{|c|}{ التاخل الثنائي بين نوعية الماء وطرائق التربية } \\
\hline متوسطات نوعية & \multicolumn{4}{|c|}{ طرائق التربية } & \multirow{2}{*}{\multicolumn{2}{|c|}{ نوعية الماء }} \\
\hline الماء & T3 & T2 & T1 & T0 & & \\
\hline 610.17 & 575.79 & 663.66 & 552.22 & 649.02 & \multirow{2}{*}{\multicolumn{2}{|c|}{ W0 }} \\
\hline $\mathbf{A}$ & $\mathbf{a}$ & $\mathbf{a}$ & $\mathbf{a}$ & $\mathbf{a}$ & & \\
\hline 547.33 & 560.71 & $\mathbf{5 1 7 . 5 7}$ & 567.21 & 543.81 & \multirow{2}{*}{\multicolumn{2}{|c|}{ W1 }} \\
\hline A & $\mathbf{a}$ & $\mathbf{a}$ & $\mathbf{a}$ & $\mathbf{a}$ & & \\
\hline
\end{tabular}

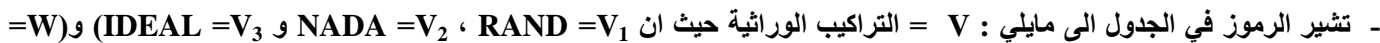

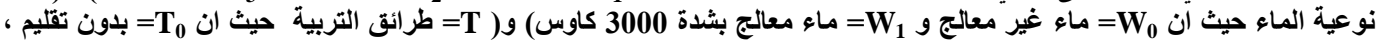

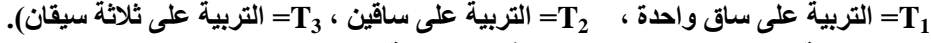
ـ القيم المتبوعة بنفس الحرف لا يوجد بينها فروق معنوية طبقا لاختبار دنكن متعدد الحدود. 
ولم يؤثرالماء المعالج مغناطيسياً معنوياً في عدد الايام

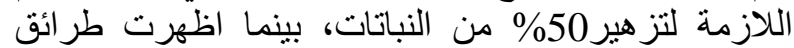

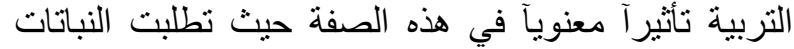

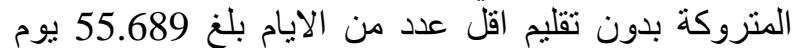
بينما تطلبت النباتات المرباة على ساقين اكبر عدد من الايان لئ الايام

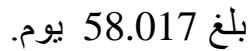

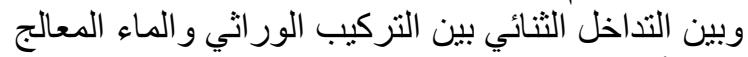

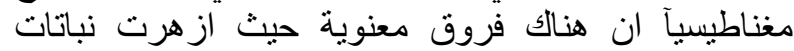

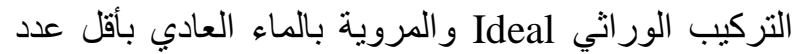

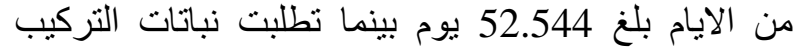

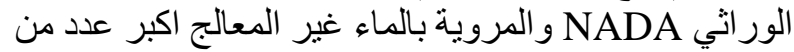

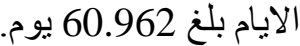
اما بالنسبة للتداخل بين التركيب الور اثي وطر ائق التربية فقد الترائ

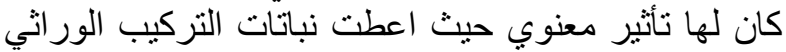
Ideal 48.597 يوم بينما استغرقت نفي نباتات التركيب الوراني التئي

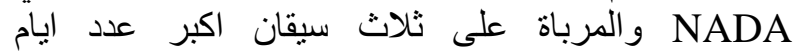

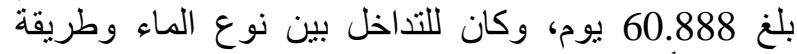
التربية تأثير معنوي حيث تطلبت النباتات المروية بالماء

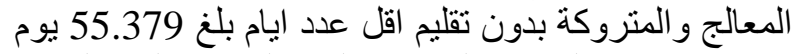

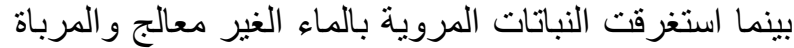

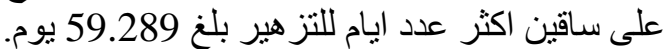

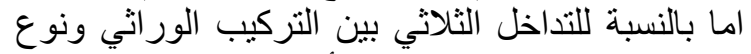

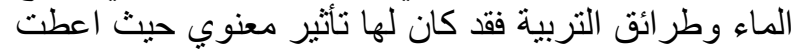

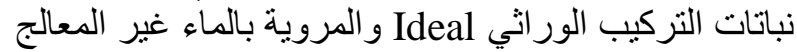

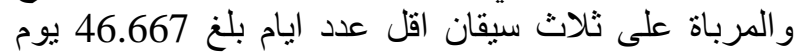

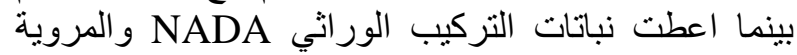
بالماء غير المعالج و المرباة على ثلاثلت سيقان اكبر عدد ايام

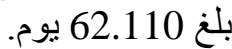

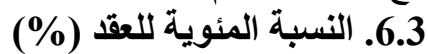

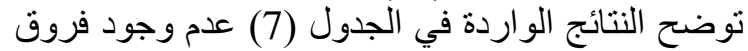

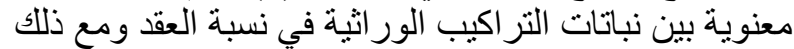

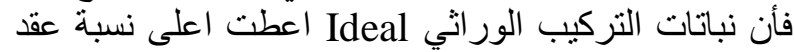

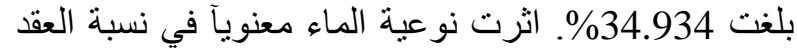

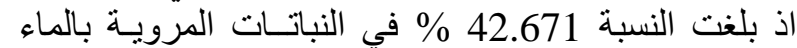

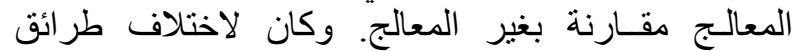

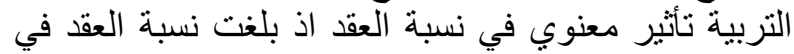

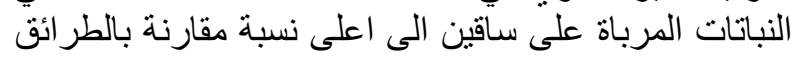
الاخرى بلغت 39.251 \%

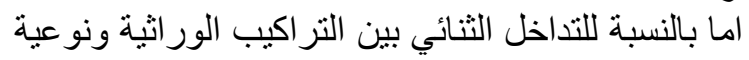

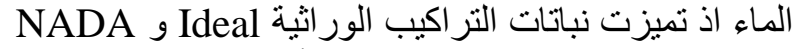

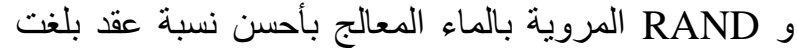

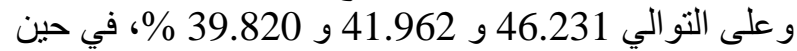
اعطت نباتات التركيب الور اثي RAND و المروية بالماء

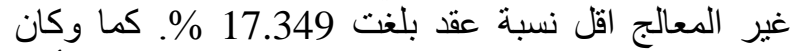
للتداخل الثنائي بين التراكيب الور اثنية وطر ائق النتربية تأثنير

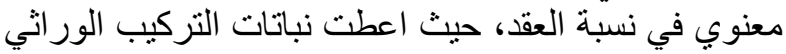

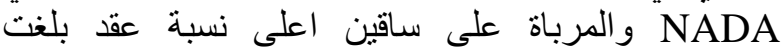

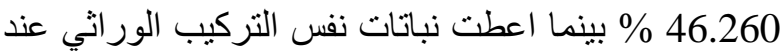

تربيتها على ثلاثة سيقان اقل نسبة عقد بلغت 260 \% 23.309 \%
محتوى الاوراق من الكلوروفيل (SPAD) :

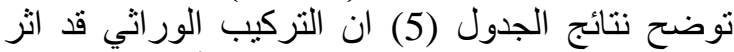

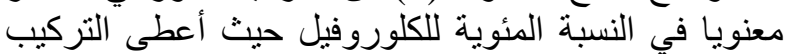

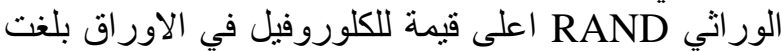

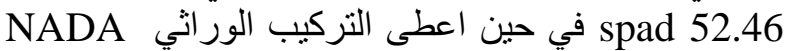

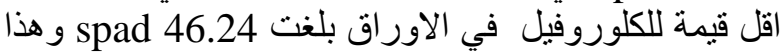

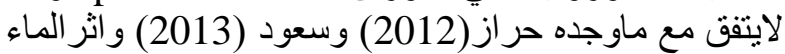

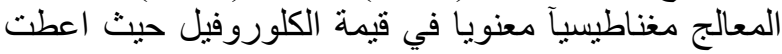
النباتات المروية بالماء المعالج مغناطيسياً بشدة 3000

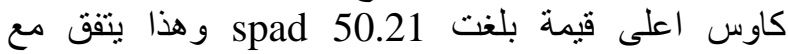
ماتوصل اليه الطبقجلي (2013) و لإنة لايتفق مع ماوجده عبيد

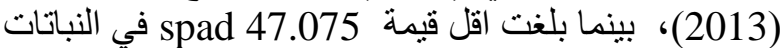
المروية بالماء غير المعالج ولم تؤثرطر ائق التربية معنويا

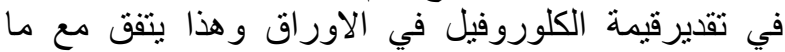
توصل اليه سعود(2013). ويبين الجدول فيلمن نفسه التداخلات

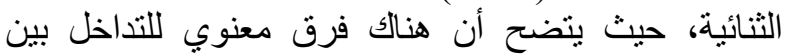

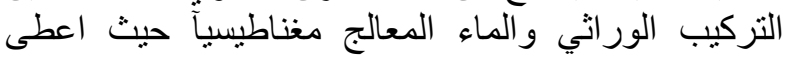

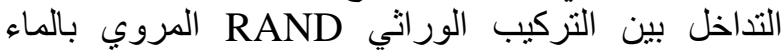
المعالج بشدة 3000 كاوس أعلى قيمة بلغت التركي 53.083

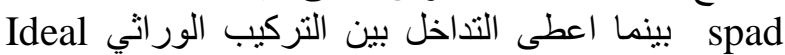

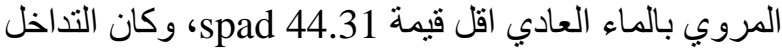
بين التركيب الوراثي وطر ائق التربية ذو تأثير معنوي في

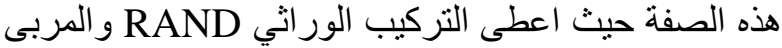

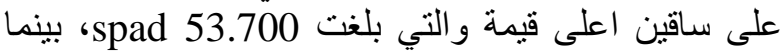
اعطى التركيب الور اثي NADA و المربي على على ساق واني واحدة

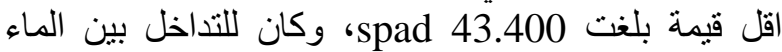

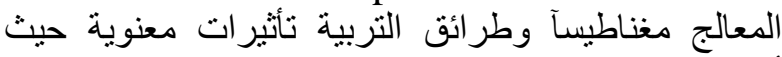

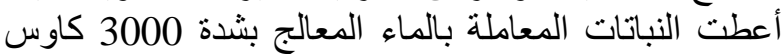

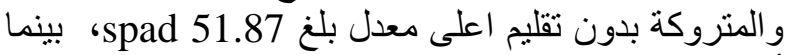

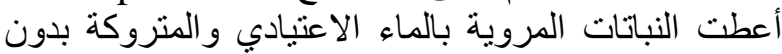

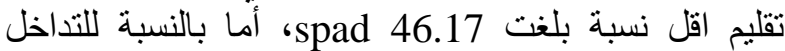

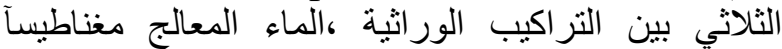

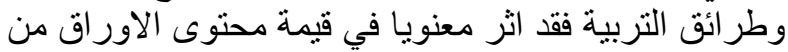
الكلوروفيل اذ سجلت نباتات التركيب الوراثي

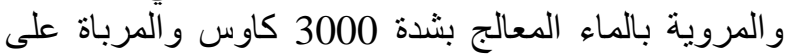

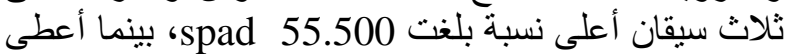

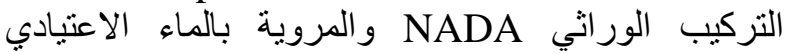
و المرباة على ساق واحدة اقل معدل 5.3. عدد الايام اللازمة لتقتح اول زهرة في 50\% من

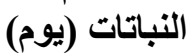

توضح نتائج الجدول (6) ان التركيب الور اثي اثر معنوياً

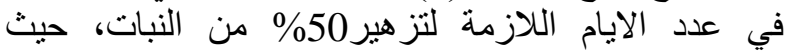

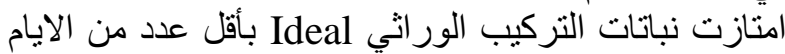
لغرض التزهيربلغ 51.689 يوم في حين نطلب التب التركيب التركيب

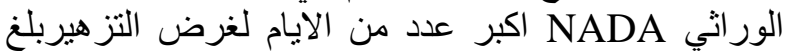
59.728 يوم ويحدث التز هير عندما يكون النبات كمية كافية النية من المادة الجافة او هرمون الفلورجين المسؤول عن التزئ هير التير

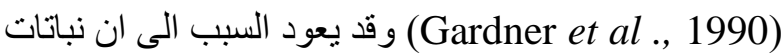

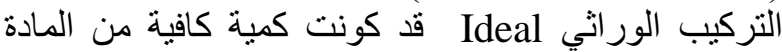
الجافة او هرمون الفلورجين قبل التراكيب الور اثية الاخرى. 
جدول (5) : تأثير التركيب الوراثي والماء المعالج مغناطيسيآوطرائق التربية وتداخلاتها في محتوى الاوراق

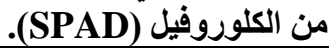

\begin{tabular}{|c|c|c|c|c|c|c|}
\hline \multirow[t]{2}{*}{ V×W التداخل V×W } & \multicolumn{4}{|c|}{ طرائق التربية } & \multirow{2}{*}{ نوعية } & \multirow{2}{*}{ التر اكيب } \\
\hline & T3 & $\mathbf{T} 2$ & T1 & T0 & & \\
\hline $\begin{array}{c}\text { 51-842 } \\
\text { a }\end{array}$ & $\begin{array}{c}51.633 \\
\text { a-e }\end{array}$ & $\begin{array}{c}54.300 \\
\text { ab }\end{array}$ & $\begin{array}{c}52.033 \\
\text { a-d }\end{array}$ & $\begin{array}{c}49.400 \\
a-f\end{array}$ & W0 & \multirow{2}{*}{ V 1} \\
\hline $\begin{array}{c}\mathbf{5 3 . 0 8 3} \\
\text { a }\end{array}$ & $\begin{array}{c}55.500 \\
\text { a }\end{array}$ & $\begin{array}{c}53.100 \\
\text { abc }\end{array}$ & $\begin{array}{c}49.400 \\
\text { a-f }\end{array}$ & $\begin{array}{c}54.333 \\
\text { ab }\end{array}$ & W1 & \\
\hline $\begin{array}{c}45.067 \\
\text { c }\end{array}$ & $\begin{array}{c}46.333 \\
\text { b-f }\end{array}$ & $\begin{array}{c}46.00 \\
\text { c-f }\end{array}$ & $\begin{array}{c}42.500 \\
f\end{array}$ & $\begin{array}{c}45.433 \\
\text { c-f }\end{array}$ & Wo & \multirow[b]{2}{*}{ V 2} \\
\hline $\begin{array}{c}47.417 \\
\text { bc }\end{array}$ & $\begin{array}{c}48.767 \\
\text { a-f }\end{array}$ & $\begin{array}{c}49.833 \\
\text { a-f }\end{array}$ & $\begin{array}{c}44.300 \\
\text { def }\end{array}$ & $\begin{array}{c}46.767 \\
\text { b-f }\end{array}$ & W1 & \\
\hline $\begin{array}{c}44.317 \\
\text { c }\end{array}$ & $\begin{array}{c}42.933 \\
\mathrm{f}\end{array}$ & $\begin{array}{c}44.133 \\
\text { def }\end{array}$ & $\begin{array}{c}46.500 \\
\text { b-f }\end{array}$ & $\begin{array}{c}43.700 \\
\text { ef }\end{array}$ & W0 & \\
\hline \multirow[t]{2}{*}{$\begin{array}{c}50.150 \\
\text { a-b }\end{array}$} & $\begin{array}{c}48.033 \\
\text { a-f }\end{array}$ & $\begin{array}{c}47.933 \\
\text { a-f }\end{array}$ & $\begin{array}{c}50.100 \\
\text { a-f }\end{array}$ & $\begin{array}{c}54.533 \\
\text { ab }\end{array}$ & W1 & V 3 \\
\hline & $\begin{array}{c}48.867 \\
\text { A }\end{array}$ & $\begin{array}{c}49.217 \\
\mathrm{~A}\end{array}$ & $\begin{array}{c}47.472 \\
\mathrm{~A}\end{array}$ & $\begin{array}{c}49.028 \\
\text { A }\end{array}$ & \multicolumn{2}{|c|}{ متوسطات طرائق التربية } \\
\hline \multicolumn{7}{|c|}{ التداخل الثنائي بين التراكيب الوراثية وطرائق التربية } \\
\hline متوسطات التراكيب & \multicolumn{4}{|c|}{ طرائق التربية } & \multirow{2}{*}{\multicolumn{2}{|c|}{ التراكيب الوراثية }} \\
\hline الوراثية & T3 & $\mathbf{T 2}$ & T1 & T0 & & \\
\hline $\begin{array}{c}52.463 \\
\mathrm{~A}\end{array}$ & $\begin{array}{c}\mathbf{5 3 . 5 6 7} \\
\text { a }\end{array}$ & $\begin{array}{c}53.700 \\
\text { a }\end{array}$ & $\begin{array}{c}\mathbf{5 0 . 7 1 7} \\
\text { abc }\end{array}$ & $\begin{array}{c}51.867 \\
\text { ab }\end{array}$ & \multicolumn{2}{|c|}{ V1 } \\
\hline $\begin{array}{c}46.242 \\
\text { В }\end{array}$ & $\begin{array}{c}47.550 \\
\text { bcd }\end{array}$ & $\begin{array}{c}47.917 \\
\text { bcd }\end{array}$ & $\begin{array}{c}43.400 \\
d\end{array}$ & $\begin{array}{c}46.100 \\
\text { cd }\end{array}$ & \multicolumn{2}{|c|}{ V2 } \\
\hline $\begin{array}{c}47.233 \\
\text { AB }\end{array}$ & $\begin{array}{c}45.483 \\
\text { cd }\end{array}$ & $\begin{array}{c}46.033 \\
\text { cd }\end{array}$ & $\begin{array}{c}48.300 \\
\text { a-d }\end{array}$ & $\begin{array}{c}49.117 \\
\text { abc }\end{array}$ & \multicolumn{2}{|c|}{$\mathbf{V 3}$} \\
\hline \multicolumn{7}{|c|}{ التذاخل الثنائي بين نوعية الماء وطرائق التربية } \\
\hline متوسطات نوعية & \multicolumn{4}{|c|}{ طرائق التربية } & \multicolumn{2}{|c|}{ 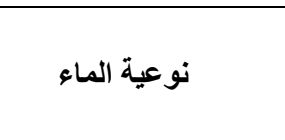 } \\
\hline $\begin{array}{c}47.075 \\
\text { B }\end{array}$ & $\begin{array}{c}46.967 \\
\text { bc }\end{array}$ & $\begin{array}{c}48.144 \\
\text { abc }\end{array}$ & $\begin{array}{c}47.011 \\
\text { bc }\end{array}$ & $\begin{array}{c}46.178 \\
c\end{array}$ & \multicolumn{2}{|c|}{ Wo } \\
\hline $\begin{array}{c}50.216 \\
\mathrm{~A}\end{array}$ & $\begin{array}{c}50.767 \\
\text { ab }\end{array}$ & $\begin{array}{c}50.289 \\
\text { abc }\end{array}$ & $\begin{array}{c}47.933 \\
\text { abc }\end{array}$ & $\begin{array}{c}51.878 \\
\text { a }\end{array}$ & \multicolumn{2}{|c|}{ W1 } \\
\hline
\end{tabular}

- تثير الرموز في الجدول الى مايلي : التراكيب الوراثية حيث ان نوعية الماء حيث أن

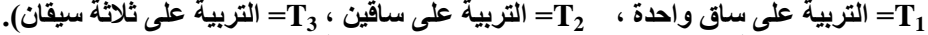

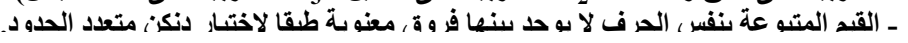


جدول (6) :تأثير التركيب الوراثي و الماء المعالج مغناطيسيا وطرائق التربية وتداخلاتها في عدد الايام لحين تزهير 50\%

\begin{tabular}{|c|c|c|c|c|c|c|}
\hline \multirow{3}{*}{ التّاخل $\mathbf{V} \times \mathbf{W}$} & & & & & \multicolumn{2}{|c|}{ من النباتات (يوم). } \\
\hline & \multicolumn{4}{|c|}{ طرائق التربية } & \multirow[t]{2}{*}{ نوعية الماء } & \multirow{2}{*}{ الوراثيةٍ } \\
\hline & T3 & T2 & T1 & T0 & & \\
\hline 60.606 & 61.333 & 62.090 & 59.667 & 59.333 & W0 & \multirow[t]{4}{*}{ V1 } \\
\hline $\mathbf{a}$ & $\mathbf{a}$ & $\mathbf{a}$ & ab & abc & & \\
\hline 57.103 & 59.443 & 57.487 & 53.260 & 58.220 & W1 & \\
\hline b & ab & a-d & def & a-d & & \\
\hline 60.972 & 62.110 & 61.110 & 60.667 & 60.00 & W0 & \multirow{4}{*}{$\mathbf{V 2}$} \\
\hline $\mathbf{a}$ & $\mathbf{a}$ & $\mathbf{a}$ & $\mathbf{a}$ & $\mathbf{a b}$ & & \\
\hline $\mathbf{5 8 . 4 8 5}$ & 59.667 & 59.440 & 59.667 & 58.167 & W1 & \\
\hline ab & ab & a-d & ab & a-d & & \\
\hline 50.833 & 46.667 & 54.667 & 53.333 & 48.667 & W0 & \multirow[t]{4}{*}{ V3 } \\
\hline c & $\mathbf{g}$ & b-e & def & fg & & \\
\hline 52.544 & $\mathbf{5 0 . 5 2 7}$ & 56.310 & $\mathbf{5 3 . 5 9 0}$ & 49.750 & W1 & \\
\hline c & efg & a-d & c-f & efg & & \\
\hline & 56.624 & 58.017 & 56.697 & 55.689 & \multirow{2}{*}{\multicolumn{2}{|c|}{ متوسطات طرائق التربية }} \\
\hline & $\mathbf{A B}$ & $\mathbf{A}$ & $\mathbf{A B}$ & B & & \\
\hline \multicolumn{7}{|c|}{ التداخل الثنائي بين التراكيب الوراثية وطرائق التربية } \\
\hline متوسطات التراكيب & \multicolumn{4}{|c|}{ طر ائق التربية } & \multirow{2}{*}{\multicolumn{2}{|c|}{ التراكيب الوراثية }} \\
\hline الوراثية & T3 & T2 & T1 & T0 & & \\
\hline 58.854 & 60.388 & 59.788 & 56.463 & 58.777 & \multirow{2}{*}{\multicolumn{2}{|c|}{ V1 }} \\
\hline A & ab & ab & bed & abc & & \\
\hline 59.728 & 60.888 & 58.775 & 60.167 & 59.083 & \multirow{2}{*}{\multicolumn{2}{|c|}{ V2 }} \\
\hline A & $\mathbf{a}$ & abc & ab & abc & & \\
\hline 51.689 & 48.597 & 55.488 & 53.462 & 49.208 & \multirow{2}{*}{\multicolumn{2}{|c|}{ V3 }} \\
\hline B & $\mathbf{e}$ & cd & d & $\mathbf{e}$ & & \\
\hline \multicolumn{7}{|c|}{ التداخل الثنائي بين نوعية الماء وطرائق التربية } \\
\hline متوسطات نوعية & \multicolumn{4}{|c|}{ طرائق التربية } & \multirow{2}{*}{\multicolumn{2}{|c|}{ نوعية الماء }} \\
\hline الماء - ل باء & T3 & T2 & T1 & T0 & & \\
\hline 57.470 & 56.703 & 59.289 & $\mathbf{5 7 . 8 8 9}$ & 56.00 & \multirow{2}{*}{\multicolumn{2}{|c|}{ W0 }} \\
\hline A & ab & $\mathbf{a}$ & ab & b & & \\
\hline 56.043 & 56.546 & 56.746 & 55.506 & 55.379 & \multirow{2}{*}{\multicolumn{2}{|c|}{ W1 }} \\
\hline $\mathbf{A}$ & $\mathbf{a b}$ & $\mathbf{a b}$ & b & b & & \\
\hline
\end{tabular}

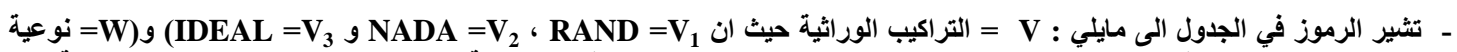

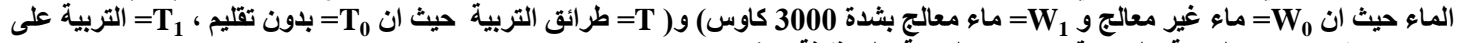

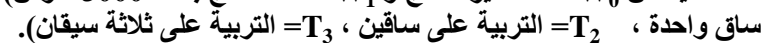
ـ القيم المتبوعة بنفس الحرف لا يوجد بينها فروق معنوية طبقا لاختبار دنكن متعدد الحدود. 


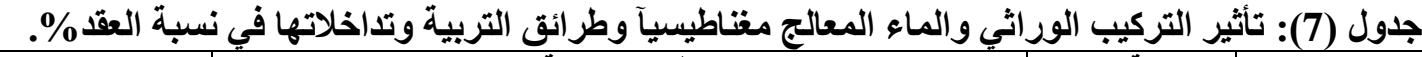

\begin{tabular}{|c|c|c|c|c|c|c|}
\hline \multirow[t]{2}{*}{ التّاخل } & \multicolumn{4}{|c|}{ طرائق التربية } & \multirow[t]{2}{*}{ نوعية الماء } & \multirow{2}{*}{ التراثية } \\
\hline & T3 & T2 & T1 & T0 & & \\
\hline 17.349 & 18.020 & 27.170 & 13.125 & 11.081 & W0 & \multirow[t]{4}{*}{ V1 } \\
\hline b & fgh & $\mathbf{c}-\mathbf{h}$ & $\mathbf{h}$ & $\mathbf{h}$ & & \\
\hline 39.820 & 33.405 & 41.333 & 42.22 & 42.319 & W1 & \\
\hline $\mathbf{a}$ & $\mathbf{c}-\mathbf{g}$ & $\mathbf{b}-\mathbf{e}$ & b - e & b - e & & \\
\hline 21.590 & 17.195 & 27.381 & 25.119 & 16.666 & W0 & \multirow[t]{4}{*}{$\mathbf{V 2}$} \\
\hline b & fgh & $\mathbf{c}-\mathbf{h}$ & $\mathbf{e}-\mathbf{h}$ & fgh & & \\
\hline 41.962 & 29.423 & 65.140 & 36.063 & 37.222 & W1 & \\
\hline $\mathbf{a}$ & $\mathbf{c}-\mathbf{h}$ & $\mathbf{a}$ & $\mathbf{c}-\mathbf{f}$ & $\mathbf{b}-\mathbf{f}$ & & \\
\hline 23.637 & 26.149 & 25.856 & 27.487 & 15.054 & W0 & \multirow[t]{4}{*}{$\mathbf{V 3}$} \\
\hline b & $\mathbf{d}-\mathbf{h}$ & $\mathbf{d}-\mathbf{h}$ & $\mathbf{c}-\mathbf{h}$ & fgh & & \\
\hline 46.231 & 56.282 & 48.624 & 47.685 & 32.335 & W1 & \\
\hline \multirow[t]{3}{*}{$\mathbf{a}$} & ab & abc & bed & $\mathbf{c}-\mathbf{h}$ & & \\
\hline & 30.079 & 39.251 & 31.950 & 25.780 & \multirow{2}{*}{\multicolumn{2}{|c|}{ متوسطات طرائق التربية }} \\
\hline & B & $\mathbf{A}$ & $\mathbf{A B}$ & B & & \\
\hline \multicolumn{7}{|c|}{ التداخل الثنائي بين التراكيب الوراثية وطرائق التربية } \\
\hline \multirow{2}{*}{ متوسطات التراكيب } & \multicolumn{4}{|c|}{ طرائق التربية } & \multirow{2}{*}{\multicolumn{2}{|c|}{ التراكيب الوراثية }} \\
\hline & T3 & T2 & T1 & T0 & & \\
\hline 28.585 & 25.713 & 34.252 & 27.673 & 26.700 & \multirow{2}{*}{\multicolumn{2}{|c|}{ V1 }} \\
\hline $\mathbf{A}$ & c & abc & bc & bc & & \\
\hline 31.776 & 23.309 & 46.260 & 30.591 & 26.944 & \multirow{2}{*}{\multicolumn{2}{|c|}{ V2 }} \\
\hline $\mathbf{A}$ & c & $\mathbf{a}$ & bc & bc & & \\
\hline 34.934 & 41.215 & 37.240 & 37.586 & 23.694 & \multirow{2}{*}{\multicolumn{2}{|c|}{ V3 }} \\
\hline $\mathbf{A}$ & $\mathbf{a b}$ & abc & abc & c & & \\
\hline \multicolumn{7}{|c|}{ التداخل الثنائي بين نوعية الماء وطرائق التربية } \\
\hline متوسطات نوعية & \multicolumn{4}{|c|}{ طرائق التربية } & \multirow{2}{*}{\multicolumn{2}{|c|}{ نوعية الماء }} \\
\hline الماء & T3 & $\mathbf{T 2}$ & T1 & T0 & & \\
\hline 20.859 & 20.455 & 26.802 & 21.910 & 14.26 & \multirow{2}{*}{\multicolumn{2}{|c|}{ W0 }} \\
\hline B & de & cd & de & $\mathbf{e}$ & & \\
\hline 42.671 & 39.703 & 51.699 & 41.990 & 37.292 & \multirow{2}{*}{\multicolumn{2}{|c|}{ W1 }} \\
\hline $\mathbf{A}$ & b & $\mathbf{a}$ & $\mathbf{a b}$ & bc & & \\
\hline
\end{tabular}

- تثير الرموز في الجدول الى مايلي : $=$ = التراكيب الوراثية حيث ان الماء حيث ان

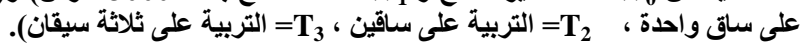
ـ القيم المتبوعة بنفس الحرف لا يوجد بينها فروق معنوية طبقا لاختبار دنكن متعدد الحدود. 
الثمس. مجلة العلوم الزر اعية العر اقية. 36 (1) : 23 . $28-$

حسين، ايمان محمود. (2002) ـ استنباط هجن فردية من ون ولئ الخيار وتقدير قوة الهجين وبعض المبان المعالم الوراثية

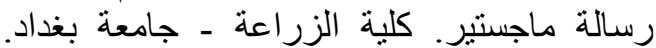

وز ارة التعليم العالي والبحث العلمي. العر اق.

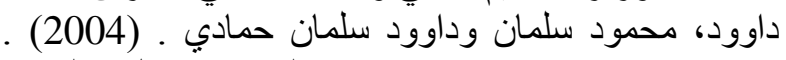

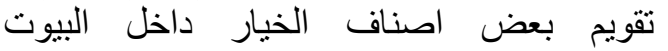
الزجاجية، مجلة الزر اعة العر اقية ، 9 (1) : 71 78

الدوري، زينب اياد عمر رزوقي. (2010). تأثير طريقة

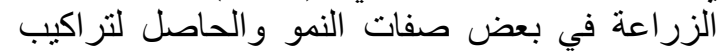

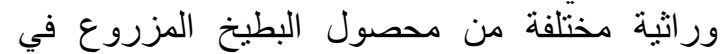
الترب الجبسية ، رسالة ماجستير ، قسم البستنة، كلية الزي، الزعة - جامعة تكريت.

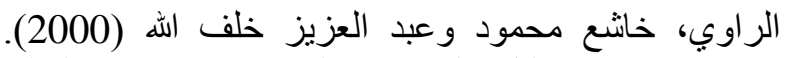

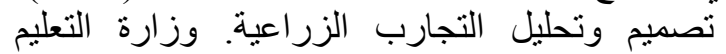
العالي و البحث العلمي، جامعة الموصل. العبل العراق.

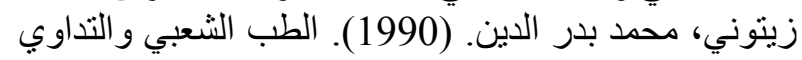
بالاعثاب. دمشق. سوريا.

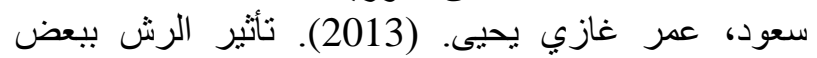

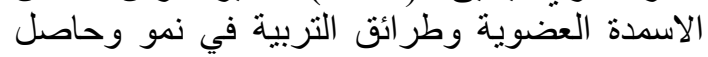

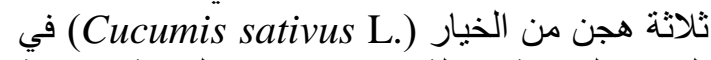
البيوت المحمية.رسالة ماجستير. قسم البستنة و هندسة

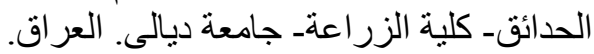

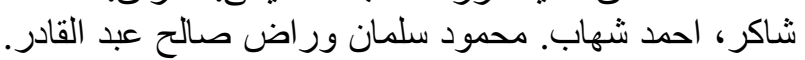

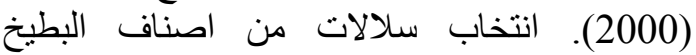
المحلي. مجلة الزراعة العراقية. مجلد (5)عدد (7)

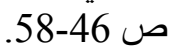

الشمري، عزيز مهدي عبد وعمر وعر غازي يحيى سعود. (2013). تأثير بعضي بعض المغذيات العضوية وبئ وطريقة التربية في نمو وحاصل ثلاثثة هجن من الخيار تحت

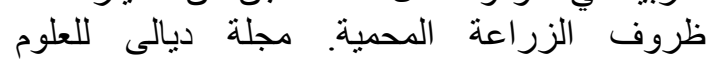
الزر اعية. 5 (2) : 283 - 294 - 294 الزية الطبقجلي، عبد الكريم عبد الجبار محمد سعيد. (2912) : 2012). تأثير

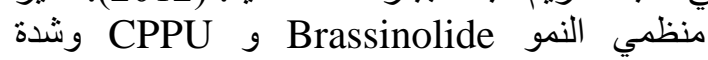
المجال المغناطيسي في نمو و إزهار صنفين من نبات المنات

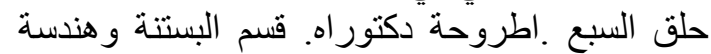

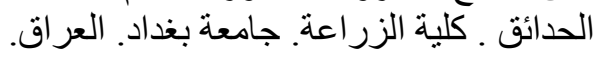

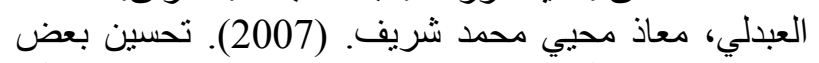
صفات البطيخ:Cucumis melo L بالانتخاب بخلية النحل. اطروحة دكتور النغاه. قسم البستنة. كلية الزراعة جامعة بغداد. العراق.

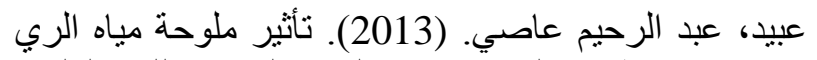

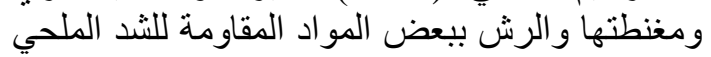
في نمو وحاصل الخبار (Cucumis sativus L.)

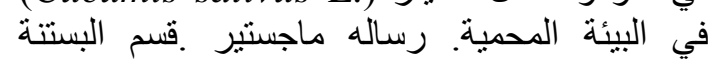

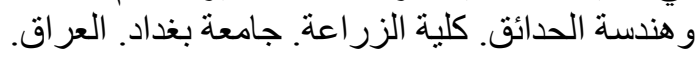

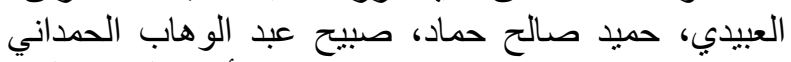
ور عد وهيب محمود. (2012). تأثير الريب الري بالماء

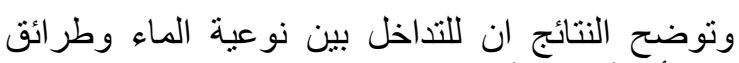

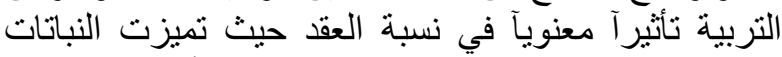

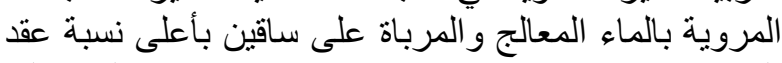

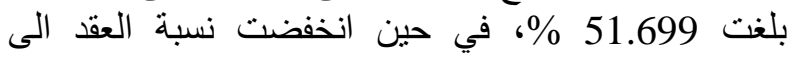
14.26 \% عند ري النباتات بالماء غير المعالج وتركها

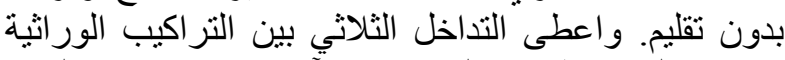

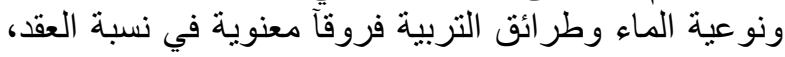

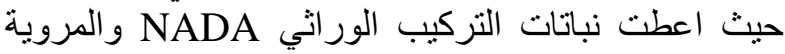

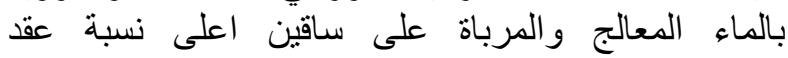

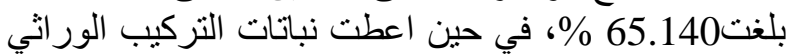
و المروية بالماء غير المعالج وغير المقلمة اقل التي نسبة عقد بلغت 11.081 \%

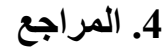

الجبوري، كاظم ديلي حسن وفيصل عبد الهادي المختار

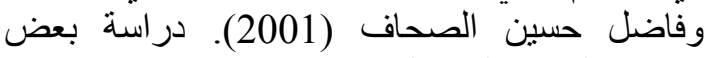

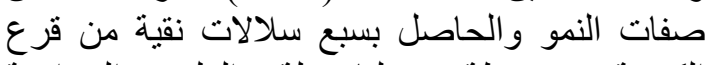

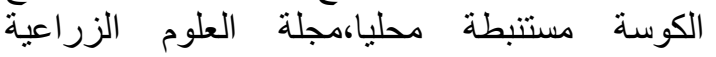

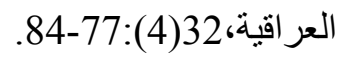

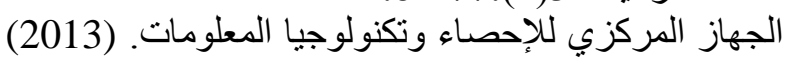

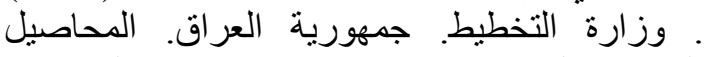

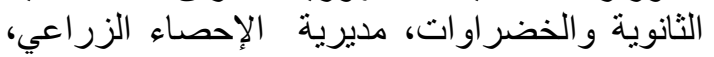
وز ارة التخطيط والتعاون الإنمائي، العر اق - بغداد.

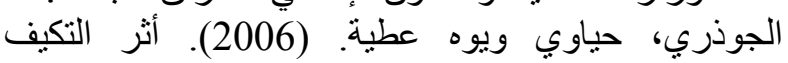

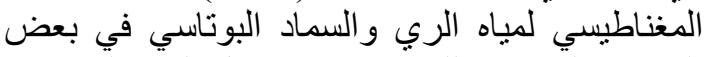

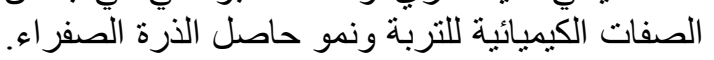

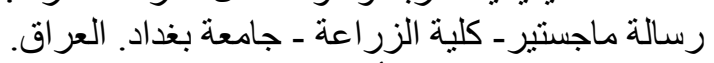

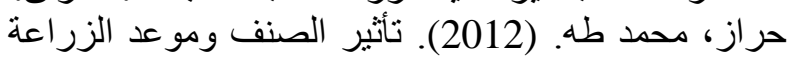

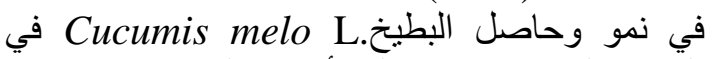

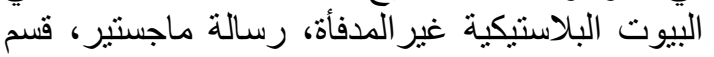

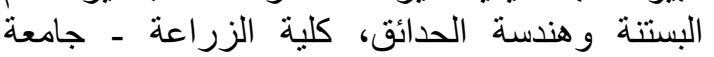
تكريت ـ العر اق.

الحربي، عبد العزيز رابح و عبد الله عبد الرحمن السعدون وصفوت عثمان خليل. (1996). تأثير طريبر طيقة التربية

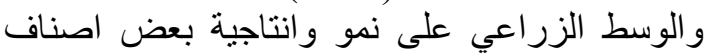

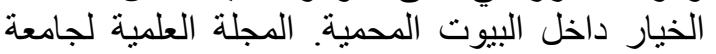

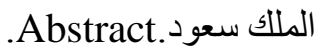

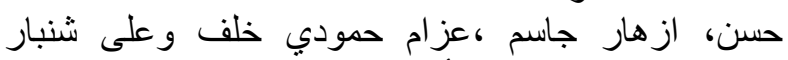

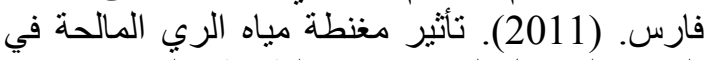

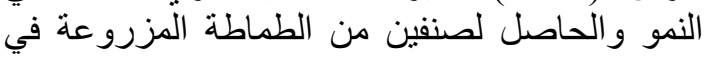

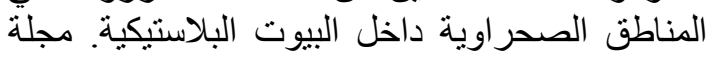

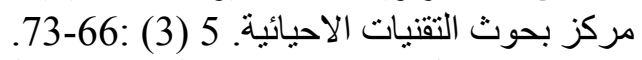

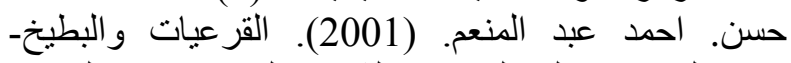

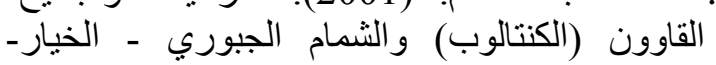

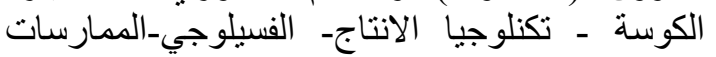

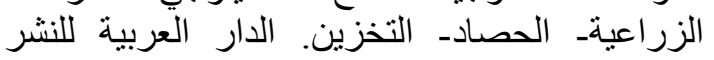
والتوزيع - القاهرة- جمهورية مصر العربة العربية.

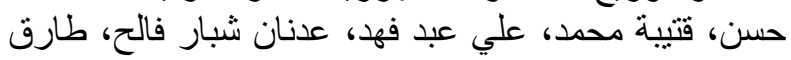

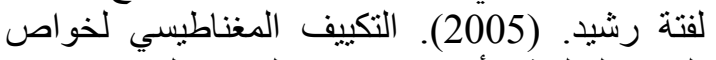
المياه المالحة لأغراض ري الكيف المحاصيل 1. زهرة لخطي 


\section{REFERENCES}

Allard R.W. (1960). Principles of plant breeding. John Wiley, New York . U.S.A.

Al Sadon A . A., Wahb - alla M. A. and Khalil S. O. (2004). Growth yield and quality of three greenhouse cucumber cultivars relation to type of water applied at different stages of plant growth. International conf. on water Resources and Arid Environment . King Saudi University, Saudi Arabia.

Gardner F. P., Vall R., and Cold D. Mc. (1990). Yield characteristics of ancient races of maiz compared to modern hybrid. Agron. J .82:862-868.

Guo F.C., Fujime Y., Hirose T. and Kato T. (1991). Effects of the number of training shoots, raising period of seedlings and Planting density In growth, fruiting and yields of sweet. pepper. J. Japan. Soc. Hort. Sci., 59: $763-770$.

Herodiza G. (1999). Observation result about the effect of magnetic tools / a series of Magnetotron size 1 - Made by Magnetic Technologies LLC - Unto the growth of consumption plant and vegetable horticulture , Collection of state documents its translation on Application technologies in different branches of economy Magnetic Technologies (L.L.C) Dubai , U.A.E.

Kronenberg K. (2005). Magneto 2 hydrodynamics: The effect of magnets on fluids GMX international. E-mail:corporate @ gmxinterhatinal. Com.

Lower R. L. and Edwards M.D. (1986). Breeding Vegetable Crops. AW publishing CO .Inc. West port, Connecticut, U.S.A.

Institute Inc. (2001). The SAS system for windows. Release, SAS 8.02. SAS inst., Cary, N.C.

Takahashi H. and Sasaki S. (1981). Studies on the Iateral shoot utilization of tomatoes 1Remarks on semi - forcing cultivation Bull. Akita. Pref . Coll. Agri.,7: 45 - 59.

Wallace B., Ebel R.C. and Kemble J. (2000). Imidacloprid effect on root growth, photosynthesis, and water use of cucumber in the greenhouse. Hort. Sci. 35 (5): 953.

Yang B.G., Yonghong W. and Xuewen C. L. (2007). Melon production in China. Acta. hort. Ishs 731:493-500.

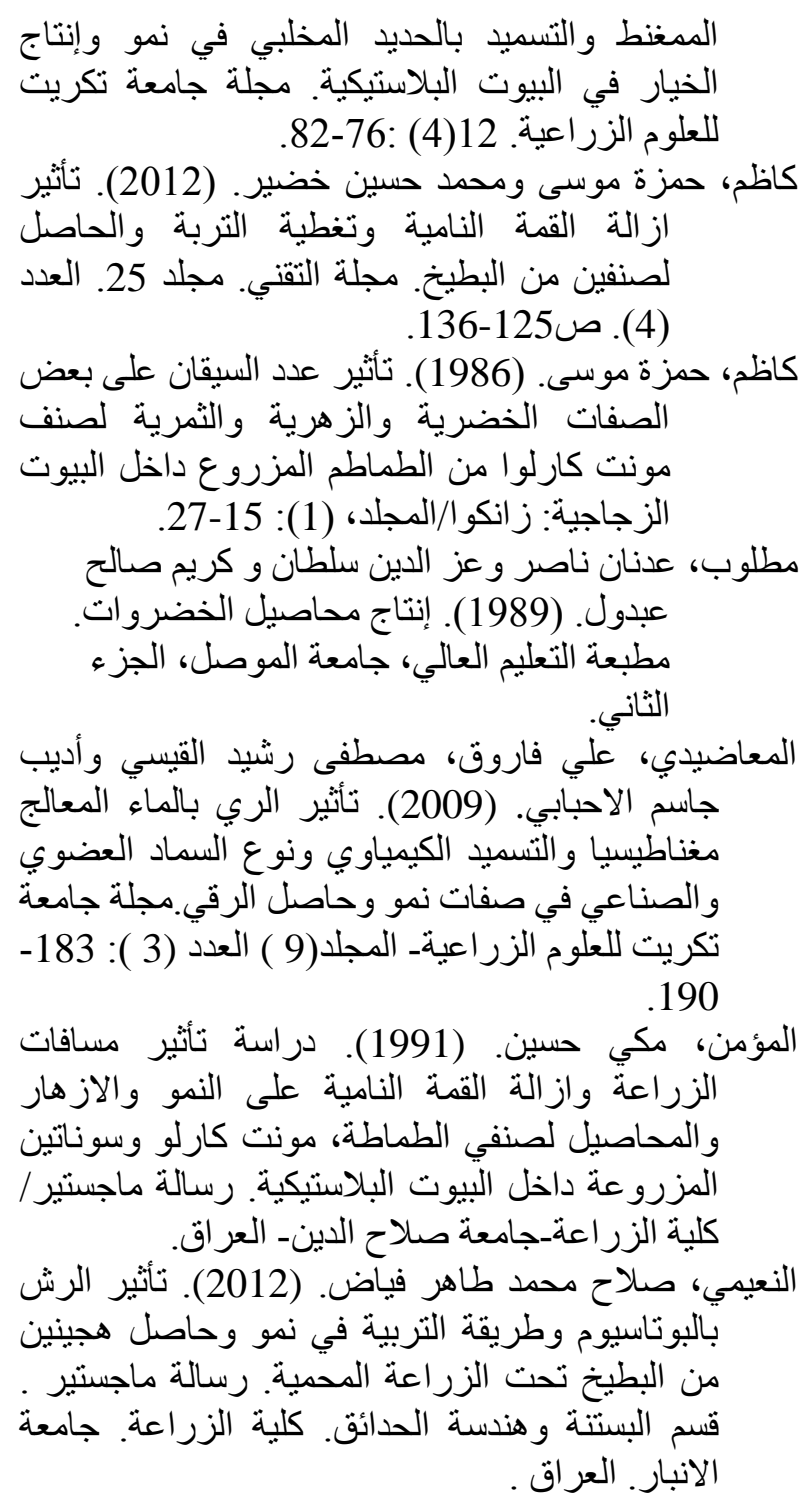

